\begin{abstract}
DO-TH 94/04
March 1994

$5 \omega 3412$

\section{Next-to-leading order hadronic single-spin production of polarized prompt photons}

\author{
W. Vogelsang \\ Institut für Physik, Universität Dortmund \\ D-44221 Dortmund, Germany

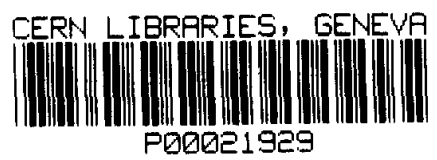

$\underline{\text { Abstract }}$

We present a calculation of the complete next-to-leading order $\left(\mathcal{O}\left(\alpha \alpha_{s}^{2}\right)\right)$ corrections to the inclusive production of polarized prompt photons in collisions of longitudinally polarized hadrons with unpolarized hadrons. We present compact analytical expressions for all our results. We show that this process should offer a good possibility to decide between presently suggested proton spin scenarios and shows good perturbative stability. Furthermore, we also estimate the effect of the leading order fragmentation contribution and discuss its influence on the cross section asymmetries. 


\section{Introduction}

The magnitude of the gluon-spin content of longitudinally polarized protons has been an issue of debate ever since the advent of the EMC result [1] on the proton's spin-dependent structure function $g_{1}^{P}$. The experimental finding of the smallness of the first moment of $g_{1}^{P}$ has been interpreted in terms of a large negative sea quark polarization $\Delta s$ in the proton [2], or of a large positive gluon polarization $\Delta G$ [3]. Unfortunately, present-day results for polarized deep-inelastic scattering do not enable us to decide between these rather different possibilities, and thus other processes need to be studied with respect to their sensitivity to $\Delta s$ and, in particular, $\Delta G$. For the latter, it is interesting to study processes in which the gluon distribution already enters in the leading order (LO), unlike the case of $g_{1}^{P}$. One of these is hadronic prompt photon production with its wellknown sensitivity to the gluon content of the incoming hadrons due to the Compton-like subprocess $q g \rightarrow \gamma q$. There are in principle two possible aspects of polarized prompt photon production. Firstly, one can consider, as is usually done, the case of two incoming longitudinally polarized hadrons, $A, B$, producing the (unpolarized) prompt photon ('twospin' case). LO $\left(\mathcal{O}\left(\alpha \alpha_{s}\right)\right)$ expectations for this process, which we refer to as $\vec{A} \vec{B} \rightarrow \gamma X$ (the arrows denoting longitudinal polarization), were presented in refs. $[4,5]$, where it was shown that this process is indeed very sensitive to the input polarized gluon distribution chosen. Quite recently, the next-to-leading order (NLO) $\left(\mathcal{O}\left(\alpha \alpha_{s}^{2}\right)\right)$ corrections to $\vec{A} \vec{B} \rightarrow$ $\gamma X$ were calculated and studied in refs. $[6,7,8]$. The main result of the study of the NLO corrections was that the process in question shows perturbative stability and thus preserves the strong sensitivity to $\Delta G$ also beyond LO [8].

Another possibility to study polarized prompt photon production is to consider the case of only one incoming hadron being longitudinally polarized, but the other being unpolarized $[9,10,5]$. In order to construct non-vanishing asymmetries in this case, it is necessary to extract more information from the final state, e.g. to determine the helicity of the outgoing prompt photon. The corresponding process is thus $\vec{A} B \rightarrow \vec{\gamma} X$. Although it might turn out to be a very hard task to experimentally study this process since it requires the measurement of the prompt photon's helicity (circular polarization), it will be an interesting challenge. The principal advantage of this 'single-spin' process is obvious: It is only necessary to have one high-energy unpolarized hadron beam which can, e.g., be scattered off a polarized target. Both these ingredients have become standard nowadays and are in principle even accessible at HERA, where the unpolarized $820 \mathrm{GeV}$ proton 
beam would yield $\sqrt{S} \approx 40 \mathrm{GeV}$ in a fixed target situation. Thus the process $\vec{A} B \rightarrow \vec{\gamma} X$ could be a viable alternative if it should turn out to be too complicated to construct highenergy polarized hadron beams in the future [11]. Leading order expectations $[10,9,5]$ for the process $\vec{A} B \rightarrow \vec{\gamma} X$, based on the Compton-like and annihilation Born cross sections, look very promising. They show a strong sensitivity to $\Delta G$ in the region of negative c.m.s. rapidities with respect to the direction of the incoming polarized hadron. Furthermore, the relevant cross section asymmetries are often larger [10] than for the 'two-spin' reaction $\vec{A} \vec{B} \rightarrow \gamma X$.

In this paper we will extend the previous studies in two ways. Firtly, we will present the NLO corrections to the process $\vec{A} B \rightarrow \vec{\gamma} X$ in order to study the perturbative stability of the process and thus to examine the question whether the strong sensitivity to $\Delta G$, found in LO, remains. Secondly, we will try to estimate the influence of the fragmentation (bremsstrahlung) contribution on the asymmetries. Although we are dealing with a fixed target situation, this could be an important issue for the following reason: As mentioned above, the results of ref. [10] show asymmetries which are larger than the corresponding ones for the two-spin case. This is expected since the two-spin asymmetries are diluted by two incoming polarized parton distributions, whereas the single-spin asymmetries are only diluted once. Including the fragmentation contribution, this statement is no longer completely correct since the single-spin cross section for the fragmentation contribution is further diluted by the polarized fragmentation functions over the unpolarized ones, as we will show in more detail below. In order to reliably establish the strong sensitivity of the process $\vec{A} B \rightarrow \vec{\gamma} X$ to the polarized gluon content of the proton it is therefore necessary to make sure that including the fragmentation contribution does not diminuish the cross section asymmetries too strongly.

The remainder of this paper is organized as follows. In section 2 we will present our calculation of the NLO corrections to $\vec{A} B \rightarrow \vec{\gamma} X$. More specifically, we will recall some definitions and expressions for the cross sections and LO results in subsection 2.1. The actual calculation is presented in section 2.2. Since it closely follows the lines of our previous calculation for $\vec{A} \vec{B} \rightarrow \gamma X$ in ref. [7], we can be rather brief here. In section 3 we collect the necessary ingredients to study the LO fragmentation contribution to $\vec{A} B \rightarrow \vec{\gamma} X$. In section 4 we present our numerical results. Section 5 contains our conclusions. Finally, Appendix A contains some calculational details, and we list our complete analytical results for the NLO corrections in Appendix B. 


\section{NLO corrections}

\subsection{Cross section formulas and $\mathrm{LO}$ results}

Before going into the calculation of the NLO corrections we briefly collect the relevant cross section formulas for the helicity transfer reaction $\vec{A} B \rightarrow \vec{\gamma} X$. The quantity we will be interested in most is the initial-final asymmetry for prompt photon production

$$
A_{\gamma}^{i f} \equiv \frac{E_{\gamma} d^{3} \delta \sigma^{A B} / d^{3} p_{\gamma}}{E_{\gamma} d^{3} \sigma^{A B} / d^{3} p_{\gamma}}
$$

where [12]

$$
E_{\gamma} \frac{d^{3} \delta \sigma^{A B}}{d^{3} p_{\gamma}} \equiv E_{\gamma} \frac{d^{3} \sigma^{A B}(++)}{d^{3} p_{\gamma}}-E_{\gamma} \frac{d^{3} \sigma^{A B}(+-)}{d^{3} p_{\gamma}},
$$

$E_{\gamma} d^{3} \sigma^{A B}\left(h_{A} \lambda_{\gamma}\right) / d^{3} p_{\gamma}$ denoting the invariant cross section for the production of a polarized prompt photon with helicity $\lambda_{\gamma}$, energy $E_{\gamma}$ and three-momentum $\vec{p}_{\gamma}$ by a polarized hadron $\vec{A}$ with helicity $h_{A}$ and an unpolarized hadron $B$. We have used in eq. (1) the ' $\delta$ ' instead of the (in spin physics often used) ' $\Delta$ ' for the cross section differences in order to indicate that we are dealing with a helicity transfer cross section and not with two incoming polarized particles. Introducing the polarized parton distributions $\Delta f_{a}^{A}\left(x, M^{2}\right)$ by

$$
\Delta f_{a}^{A}\left(x, M^{2}\right) \equiv\left(f_{a}^{A}\right)_{+}\left(x, M^{2}\right)-\left(f_{a}^{A}\right)_{-}\left(x, M^{2}\right)
$$

where $\left(f_{a}^{A}\right)_{+(-)}\left(x, M^{2}\right)$ denotes the distribution of parton type $a$ with positive (negative) helicity in hadron $A$ with positive helicity at scale $M^{2}$, we can relate the hadronic and the subprocess cross sections [13]:

$E_{\gamma} \frac{d^{3} \delta \sigma^{A B}}{d^{3} p_{\gamma}}=\frac{1}{\pi p_{T}^{4}} \sum_{a, b} \int_{\frac{x_{T}}{2} e^{\eta}}^{1-\frac{x_{T}}{2} e^{-\eta}} d v \int_{\frac{x_{T}}{2} e^{\eta} / v}^{1} d w x_{1} \Delta f_{a}^{A}\left(x_{1}, M^{2}\right) x_{2} f_{b}^{B}\left(x_{2}, M^{2}\right) v w(1-v) \hat{s} \frac{d \delta \hat{\sigma}^{a b}}{d v d w}$,

where $\sum_{a, b}$ denotes the sum over the appropriate parton combinations. Here we have introduced the prompt photon's transverse momentum $p_{T}$ and its rapidity $\eta$ in the c.m.s. of the colliding hadrons, measured in the direction of the polarized hadron $A$. Furthermore we have defined

$$
\begin{aligned}
v & \equiv 1+\frac{\hat{t}_{1}}{\hat{s}} \\
w & \equiv \frac{-\hat{u}_{1}}{\hat{s}+\hat{t}_{1}}
\end{aligned}
$$


with $\hat{t}_{1}=\left(x_{1} P_{A}-p_{\gamma}\right)^{2}, \hat{u}_{1}=\left(x_{2} P_{B}-p_{\gamma}\right)^{2}$ and $\hat{s}=\left(x_{1} P_{A}+x_{2} P_{B}\right)^{2}=x_{1} x_{2} S, P_{A}$ and $P_{B}$ being the momenta of the incoming hadrons. In terms of $v$ and $w$ we have $x_{T} \equiv 2 p_{T} / \sqrt{S}$, $x_{1}=x_{T} e^{\eta} / 2 v w$ and $x_{2}=x_{T} e^{-\eta} / 2(1-v)$. The helicity transfer subprocess cross sections $d \delta \hat{\sigma}^{a b} / d v d w$ in eq. (4) are defined in complete analogy with eq. (2), the ' $\delta$ ' now referring to the difference of cross sections for definite helicities of parton $a$ and the prompt photon. Finally, $f_{b}^{B}\left(x, M^{2}\right)$ is the usual unpolarized distribution of parton $b$ in hadron $B$.

In $\mathrm{LO}$ we have $\hat{s}+\hat{t}_{1}+\hat{u}_{1}=0$, which is equivalent to $w=1$. In fact,

$$
\frac{d \hat{\sigma}_{2 \rightarrow 2}^{a b}}{d v d w}=\frac{d \hat{\sigma}_{2 \rightarrow 2}^{a b}}{d v} \delta(1-w)
$$

The contributing subprocesses are $\vec{q} \vec{q} \rightarrow \vec{\gamma} g, \vec{q} g \rightarrow \vec{\gamma} q$ and $\vec{g} q \rightarrow \vec{\gamma} q$, the subprocess cross sections for which read $[9,10,5]$ :

$$
\begin{aligned}
\frac{d \delta \hat{\sigma}}{d v}(\vec{q} \vec{q} \rightarrow \vec{\gamma} g) & =\frac{d \delta \hat{\sigma}}{d v}(\overrightarrow{\vec{q}} q \rightarrow \vec{\gamma} g) \\
& =\frac{2 C_{F}}{N_{C}} \frac{\pi \alpha \alpha_{s} e_{q}^{2}}{\hat{s}} \frac{v^{2}-(1-v)^{2}}{v(1-v)}, \\
\frac{d \delta \hat{\sigma}}{d v}(\vec{q} g \rightarrow \vec{\gamma} q) & =\frac{1}{N_{C}} \frac{\pi \alpha \alpha_{s} e_{q}^{2}}{\hat{s}} \frac{1-(1-v)^{2}}{1-v}, \\
\frac{d \delta \hat{\sigma}}{d v}(\vec{g} q \rightarrow \vec{\gamma} q) & =\frac{1}{N_{C}} \frac{\pi \alpha \alpha_{s} e_{q}^{2}}{\hat{s}} \frac{1+v^{2}}{v}
\end{aligned}
$$

where $N_{C}$ is the number of colors, $N_{C}=3$, and $C_{F}=\left(N_{C}^{2}-1\right) / 2 N_{C}$. The last subprocess, eq. (9), generates the sensitivity of $\vec{A} B \rightarrow \vec{\gamma} X$ to the polarized gluon distribution of hadron $A$. Let us note that the corresponding unpolarized results, needed in the denominator of $A_{\gamma}^{i f}$ in eq. (1), can be obtained by removing all ' $\delta$ 's and ' $\Delta$ 's and using the unpolarized subprocess cross sections (see e.g. [7]) instead of the polarized ones.

\subsection{Calculation of the NLO corrections}

The strategy for calcluating the NLO $\left(\mathcal{O}\left(\alpha \alpha_{s}^{2}\right)\right)$ corrections to the process $\vec{A} \vec{B} \rightarrow \gamma X$ was already presented in much detail in ref. [7]. Therefore we only have to describe here the main steps of the calculation for the corresponding corrections to $\vec{A} B \rightarrow \vec{\gamma} X$ and discuss the differences to our previous calculation wherever necessary. The processes we have to calculate are $q \bar{q} \rightarrow \gamma g$ and $q g \rightarrow \gamma q$ (virtual corrections), $q \bar{q} \rightarrow \gamma g g, q g \rightarrow \gamma q g, q \bar{q} \rightarrow \gamma q^{\prime} \bar{q}^{\prime}$, $g g \rightarrow \gamma q \bar{q}, q q \rightarrow \gamma q q, q \bar{q} \rightarrow \gamma q \bar{q}$ and $q q^{\prime} \rightarrow \gamma q q^{\prime}$ (for the corresponding Feynman graphs see ref. [7]), where we have to take into consideration that the outgoing photon and one 
of the incoming particles are polarized. This means, e.g., that there are two contributions for $q g$ scattering, namely $\vec{q} g \rightarrow \vec{\gamma} q(g)$ and $\vec{g} q \rightarrow \vec{\gamma} q(g)$ which will have different cross sections (see also the LO results, eqs. (8), (9)). In order to regularize the various types of singularities appearing in the calculation we work in dimensional regularization. To project onto the helicity state $\lambda_{\gamma}$ of the prompt photon we use (for the $2 \rightarrow 3$ processes) the polarization tensor

$$
\epsilon_{\alpha}^{*}\left(k_{1}, \lambda_{\gamma}\right) \epsilon_{\beta}\left(k_{1}, \lambda_{\gamma}\right)=-i \lambda_{\gamma} \epsilon_{\alpha \beta \rho \sigma} \frac{k_{1}^{\rho}\left(k_{2}+k_{3}\right)^{\sigma}}{\hat{s}-s_{23}},
$$

where $k_{1}, k_{2}$ and $k_{3}$ are the momenta of the prompt photon and the other two outgoing particles, respectively, and we have

$$
s_{23}=\left(k_{2}+k_{3}\right)^{2}=\hat{s} v(1-w)
$$

Due to this projector and the projectors onto the helicity states of one of the incoming particles we encounter $\gamma_{5}$ and $\epsilon_{\mu \nu \rho \sigma}$ in the calculation. In order to consistently work with these quantities in $n=4-2 \epsilon$ dimensions we use the HVBM scheme $[14,15,16]$ which is, to present knowledge, the only internally completely consistent one. Let us note that we average the spin of incoming unpolarized gluons (which turn up, e.g., in $\vec{q} g$ scattering) by $1 / 2(1-\epsilon)$.

For the $\overline{\mathrm{MS}}$-renormalized virtual corrections to $\vec{q} \vec{q} \rightarrow \vec{\gamma} g$ (being equal to $\overrightarrow{\bar{q}} q \rightarrow \vec{\gamma} g$ ), $\vec{q} g \rightarrow \vec{\gamma} q$ and $\vec{g} q \rightarrow \vec{\gamma} q$ we find:

$$
\begin{aligned}
v(1- & v) \hat{s} \frac{d \delta \hat{\sigma}^{V}}{d v d w}(\vec{q} \bar{q} \rightarrow \vec{\gamma} g)=\frac{\alpha \alpha_{s}^{2}\left(\mu^{2}\right) e_{q}^{2} \mu^{2 \epsilon}}{\Gamma(1-2 \epsilon)}\left(\frac{\left(4 \pi \mu^{2}\right)^{2}}{\hat{t}_{1} \hat{u}_{1}}\right)^{\epsilon} \frac{C_{F}}{N_{C}} \delta(1-w)\left[-\frac{\left(2 C_{F}+N_{C}\right) \delta T_{q \bar{q}}}{\epsilon^{2}}\right. \\
& +\frac{1}{\epsilon}\left(\frac{N_{F}}{3} \delta T_{q \bar{q}}+N_{C} \delta T_{q \bar{q}}\left(\ln v_{1}+\ln v\right)+\frac{1}{6} N_{C}(17-22 v)+C_{F}(5-6 v)\right) \\
& +b_{0}+\frac{1}{6}\left(4 C_{F}-N_{C}\right) \pi^{2} \delta T_{q \bar{q}}+b_{0} \delta T_{q \bar{q}} \ln \frac{\mu^{2}}{\hat{s}}-N_{C} \delta T_{q \bar{q}} \ln v \ln v_{1}+2 C_{F}(5-7 v) \\
& +\frac{1}{2}\left(2 C_{F}-N_{C}\right)(2-v) v \ln ^{2} v_{1}+C_{F} v(2+v) \ln v_{1}-C_{F}(3-v) v_{1} \ln v \\
& \left.-\frac{1}{2}\left(2 C_{F}-N_{C}\right)(1+v) v_{1} \ln ^{2} v-N_{C}\left(v^{2}+v_{1}\right) \ln v-N_{C}\left(1+v v_{1}\right) \ln v_{1}\right], \quad(12) \\
v(1- & v) \hat{s} \frac{d \delta \hat{\sigma}^{V}}{d v d w}(\vec{q} g \rightarrow \vec{\gamma} q)=\frac{\alpha \alpha_{s}^{2}\left(\mu^{2}\right) e_{q}^{2} \mu^{2 \epsilon}}{\Gamma(1-2 \epsilon)}\left(\frac{\left(4 \pi \mu^{2}\right)^{2}}{\hat{t}_{1} \hat{u}_{1}}\right)^{\epsilon} \frac{1}{N_{C}} \delta(1-w)\left[-\frac{\left(2 C_{F}+N_{C}\right) v \delta T_{q g}}{2 \epsilon^{2}}\right. \\
+ & \frac{v}{\epsilon}\left(-\frac{1}{2} b_{0} \delta T_{q g}+\frac{1}{2} N_{C} \delta T_{q g} \ln v_{1}+\frac{1}{2}\left(2 C_{F}-N_{C}\right) \delta T_{q g} \ln v-\frac{1}{2} C_{F} v(6-5 v)+\frac{N_{C} v^{2}}{2}\right) \\
+ & \frac{1}{2} b_{0} v \delta T_{q g} \ln \frac{\mu^{2}}{\hat{s}}-\frac{1}{4}\left(2 C_{F}-N_{C}\right) v(1-2 v) \ln v_{1} \ln \frac{v_{1}}{v^{2}}-C_{F} v^{2}(7-5 v)+\frac{1}{2} b_{0} v^{3}
\end{aligned}
$$




$$
\begin{aligned}
\frac{1}{\hat{s} v} \frac{d \delta \sigma^{F}}{d v d w} & =-\frac{\alpha_{s}}{2 \pi}\left[\frac{1}{\hat{s} v} \Delta H_{q q}\left(w, M^{2}\right) \frac{d \delta \hat{\sigma}^{\vec{q} g \rightarrow \vec{\gamma} q}}{d v}(w \hat{s}, v, \epsilon)\right. \\
& +\frac{1}{\hat{s}(1-v w)} H_{q g}\left(\frac{1-v}{1-v w}, M^{2}\right) \frac{d \delta \hat{\sigma}^{\vec{q} q \rightarrow \vec{\gamma} g}}{d v}\left(\frac{1-v}{1-v w} \hat{s}, v w, \epsilon\right) \\
& \left.+\frac{1}{\hat{s}(1-v w)} H_{g g}\left(\frac{1-v}{1-v w}, M^{2}\right) \frac{d \delta \hat{\sigma}^{\vec{q} g \rightarrow \vec{\gamma} q}}{d v}\left(\frac{1-v}{1-v w} \hat{s}, v w, \epsilon\right)\right] \\
& -\frac{\alpha}{2 \pi}\left[\frac{1}{\hat{s}(1-v+v w)} \Delta H_{\gamma q}\left(1-v+v w, M^{\prime 2}\right) \frac{d \delta \hat{\sigma}^{\vec{q} g \rightarrow \vec{q} g}}{d v}\left(\hat{s}, \frac{v w}{1-v+v w}, \epsilon\right)\right] .
\end{aligned}
$$

Here the $d \delta \hat{\sigma}^{\vec{a} b \rightarrow \vec{c} d} / d v(\hat{s}, v, \epsilon)$ are the $n$-dimensional $2 \rightarrow 2$ cross sections for the processes $\vec{a} b \rightarrow \vec{c} d$ and are listed in Appendix A. For consistency, they have, of course, to be calculated in the HVBM scheme. Furthermore, we have for the initial state singularities

$$
(\Delta) H_{i j}\left(z, M^{2}\right) \equiv-\frac{1}{\hat{\epsilon}}(\Delta) P_{i j}(z)\left(\frac{\mu^{2}}{M^{2}}\right)^{\epsilon}+(\Delta) f_{i j}(z) \quad(i, j=q, g)
$$

where $1 / \hat{\epsilon} \equiv 1 / \epsilon-\gamma_{E}+\ln 4 \pi$ and $M$ is the (initial state) factorization scale. In eq. (19) the $(\Delta) P_{i j}(z)$ denote the well-known unpolarized (polarized) one-loop splitting functions for the transitions $j \rightarrow i[19]$. The functions $(\Delta) f_{i j}(z)$ represent the freedom in choosing a factorization prescription. In the $\overline{\mathrm{MS}}$ scheme (which we adopt for the unpolarized case) these functions vanish. In the second and third line of eq. (19), the unpolarized splitting terms $H_{q g}$ and $H_{g g}$ must appear since we are dealing with an unpolarized incoming gluon (for $\vec{g} q$ scattering the situation would just be reversed). The novelty (with respect to our previous calculation in which the prompt photon was unpolarized) is the presence of the polarized quark-to-photon splitting term $\Delta H_{\gamma q}\left(z, M^{\prime 2}\right)$ in eq. (18) which reads in analogy to eq. (19)

$$
\Delta H_{\gamma q}\left(z, M^{\prime 2}\right)=-\frac{1}{\hat{\epsilon}} \Delta P_{\gamma_{q}}(z)\left(\frac{\mu^{2}}{M^{\prime 2}}\right)^{\epsilon}+\Delta f_{\gamma q}(z)
$$

with

$$
\Delta P_{\gamma q}(z)=\frac{1-(1-z)^{2}}{z}
$$

This contribution corresponds to a final state singularity which has to be factorized into the polarized photon fragmentation contribution at a scale $M^{\prime}$.

We now fix the functions $(\Delta) f_{i j}$ and $\Delta f_{\gamma q}$ in order to specify our choice for the factorization scheme. For the $(\Delta) f_{i j}(i, j=q, g)$ we adopt the same choice as in our previous calculation, namely

$$
f_{i j}(z)=0
$$

for the unpolarized ones ( $\overline{\mathrm{MS}}$-scheme) and

$$
\Delta f_{i j}(z)=a_{i j}(1-z)
$$


where

$$
\begin{array}{ll}
a_{q q}=-4 C_{F}, & a_{q g}=2 T_{R}, \\
a_{g q}=-2 C_{F}, & a_{g g}=-4 N_{C} .
\end{array}
$$

for the polarized ones $\left(\overline{\mathrm{MS}}_{\mathrm{P}}\right.$-scheme). The reason for the latter choice was given in much detail in ref. [7], where we showed that the $(1-z)$-terms presented in eqs. (23), (24) have a collinear origin in the HVBM scheme and are universal for the respective collinear transition $j \rightarrow i$ in this scheme, i.e. do not depend on the subprocess under consideration. Thus it seems most plausible to subtract them along with the $1 / \hat{\epsilon}$-poles and absorb them into the definition of the polarized parton distribution in NLO [20]. This is the statement of eqs. (23), (24).

We choose to extend the $\overline{\mathrm{MS}}_{\mathrm{P}}$ scheme adopted in our previous calculation to the function $\Delta f_{\gamma q}(z)$ by setting

$$
\Delta f_{\gamma q}(z)=-2(1-z)
$$

in analogy with the expression for $\Delta f_{g q}(z)$ (eq. (24)) when the color factor is adjusted correctly. This choice is supported by the observation that in the limit when the polarized prompt photon becomes collinear with the outgoing quark in the process (e.g.) $\vec{q}\left(p_{1}\right) g\left(p_{2}\right) \rightarrow \vec{\gamma}\left(k_{1}\right) q\left(k_{2}\right) g\left(k_{3}\right)\left(k_{1}=\frac{x}{1-x} k_{2}\right.$, Fig. 1) the $2 \rightarrow 3$ matrix element reduces to

$$
\delta|M|_{\vec{q} g \rightarrow \vec{\gamma} g g}^{2} \sim(-\epsilon)\left[-\frac{1}{\epsilon} \Delta P_{\gamma q}(x)-2(1-x)\right] \delta|M|_{\vec{q} g \rightarrow \vec{q} g}^{2} .
$$

The structure of the terms in brackets is again the same for all subprocesses in the collinear limit $k_{1} \| k_{2}$ (particle 2 being a quark) and thus is universal for the transition $\vec{q} \rightarrow \vec{\gamma}$ in the HVBM scheme. It is therefore most plausible to absorb these terms into the polarized quark-to-photon fragmentation function in NLO. It should be noted that in spite of the attractiveness of the $\overline{\mathrm{MS}}_{\mathrm{P}}$ scheme any other choice is possible. Of course, physical results must be independent of the choice of the factorization scheme. Any change in the functions $(\Delta) f_{i j}(z)$ or $\Delta f_{\gamma q}(z)$ leads to a corresponding modification of the two-loop (space- or time-like) Altarelli-Parisi splitting functions in such a way that physical results remain unchanged. Unfortunately the latter functions are not known up to now in the polarized case.

Adding up the virtual, the $2 \rightarrow 3$ and the factorization cross sections, we arrive at our final result, which can be cast into the following form for all subprocesses: 


$$
\begin{aligned}
v w(1-v) \hat{s} \frac{d \delta \hat{\sigma}}{d v d w} & =\alpha \alpha_{s}^{2}\left(\mu^{2}\right)\left[\left(\delta c_{a} \delta(1-w)+\delta c_{b} \frac{1}{(1-w)_{+}}+\delta c_{c}\right) \ln \frac{M^{2}}{\hat{s}}+\delta c_{d} \ln \frac{M^{\prime 2}}{\hat{s}}\right. \\
& +\delta c_{1} \delta(1-w)+\delta c_{2} \frac{1}{(1-w)_{+}}+\delta c_{3}\left(\frac{\ln (1-w)}{1-w}\right)_{+} \\
& +\delta c_{4} \ln v+\delta c_{5} \ln (1-v)+\delta c_{6} \ln w+\delta c_{7} \frac{\ln w}{1-w}+\delta c_{8} \ln (1-w) \\
& +\delta c_{9} \ln (1-v w)+\delta c_{10} \frac{\ln \frac{1-v}{1-v w}}{1-w}+\delta c_{11} \ln (1-v+v w) \\
& \left.+\delta c_{12} \frac{\ln (1-v+v w)}{1-w}+\delta c_{13}\right]
\end{aligned}
$$

where $\mu$ is the renormalization scale, $M$ the initial-state factorization scale and $M^{\prime}$ the fragmentation scale. The coefficients $\delta c_{i}(v, w)(i=a, \ldots, d ; 1, \ldots, 13)$ for the various subprocesses are listed in Appendix B. As in our previous calculation [7] we absorb all contributions $\sim e_{q}^{2}$ from $\vec{q} \bar{q} \rightarrow \vec{\gamma} q^{\prime} \bar{q}^{\prime}$ (where $e_{q}$ is the charge of quark $q$ ) along with corresponding terms from the cross section for $\vec{q} \bar{q} \rightarrow \vec{\gamma} q \bar{q}$ into the cross section for $\vec{q} \bar{q} \rightarrow$ $\vec{\gamma} g g$ where these contributions then appear as terms $\sim N_{F}$ in $\delta c_{1}, \delta c_{2}$ and $\delta c_{13}$. We find that the final result for $\vec{q} \bar{q} \rightarrow \vec{\gamma} a b\left(a b=g g, q \bar{q}\right.$ or $\left.q^{\prime} \bar{q}^{\prime}\right)$ equals the one for $\overrightarrow{\bar{q}} q \rightarrow \vec{\gamma} a b$, thus maintaining the corresponding property of the leading order result (see eq. (7)). Finally, the cross sections for $\vec{q} \vec{q}^{\prime} \rightarrow \vec{\gamma} q \bar{q}^{\prime}$ and $\overrightarrow{\bar{q}} q^{\prime} \rightarrow \vec{\gamma} \bar{q} q^{\prime}$ can be obtained from the one for $\vec{q} q^{\prime} \rightarrow \vec{\gamma} q q^{\prime}$ by simply changing $e_{q}^{\prime} \rightarrow-e_{q}^{\prime}$, where $e_{q}^{\prime}$ is the charge of the $q^{\prime}$.

\section{LO fragmentation contribution}

As stated in the introduction, the fragmentation contribution to the process $\vec{A} B \rightarrow \vec{\gamma} X$ could be of some importance since it is likely to change the size of the asymmetry $A_{\gamma}^{i f}$ expected for the 'direct' (non-fragmenting) contributions. This can be easily seen from the expression for the polarized LO $\left(\mathcal{O}\left(\alpha \alpha_{s}\right)\right)$ fragmentation cross section:

$$
\begin{aligned}
E_{\gamma} \frac{d^{3} \delta \sigma_{f r a g}^{A B}}{d^{3} p_{\gamma}} & =\frac{1}{\pi p_{T}^{2} S} \sum_{a b c d} \int_{z_{\min }}^{1} d z \int_{\frac{x_{T}}{2 z} e^{\eta}}^{1-\frac{x_{T}}{2 z} e^{-\eta}} d v \Delta f_{a}^{A}\left(x_{1}, M^{2}\right) f_{b}^{B}\left(x_{2}, M^{2}\right) \\
& \times \frac{\hat{s} d \delta \hat{\sigma}^{\vec{a} b \rightarrow \vec{c} d}(\hat{s}, v)}{d v} \Delta D_{c}^{\gamma}\left(z, M^{\prime 2}\right)
\end{aligned}
$$

where $z_{\min } \equiv x_{T}\left(e^{\eta}+e^{-\eta}\right) / 2, x_{1}=x_{T} e^{\eta} / 2 z v, x_{2}=x_{T} e^{-\eta} / 2 z(1-v)$, and the sum correctly runs over all $2 \rightarrow 2$ helicity transfer QCD subprocesses $\vec{a} b \rightarrow \vec{c} d$ with cross sections 
$d \delta \hat{\sigma}^{\vec{a} b \rightarrow \vec{c} d}(\hat{s}, v) / d v$, most of which were already needed for the factorization of final state mass singularities in the last section and have been collected in Appendix A. For our present purpose they can be taken from this appendix, setting $\epsilon=0$ there. The additionally needed cross sections involving final state polarized gluons can be found at the end of Appendix A. In eq. (28), $\Delta D_{c}^{\gamma}\left(z, M^{\prime 2}\right)$, which is $\mathcal{O}\left(\alpha / \alpha_{s}\right)$, is the polarized distribution function at scale $M^{\prime}$ for the fragmentation of a polarized parton type $c$ into a polarized photon, defined in complete analogy with eq. (3). Adding the LO 'direct' (i.e. nonfragmenting) contribution (LO version of eq. (4)) and eq. (28), gives the full cross section for the process $\vec{A} B \rightarrow \vec{\gamma} X$. Up to now, nothing is known at all about the polarized photon fragmentation functions, apart from the necessary constraint $\left|\Delta D_{c}^{\gamma}\left(z, M^{\prime 2}\right)\right| \leq D_{c}^{\gamma}\left(z, M^{\prime 2}\right)$, where $D_{c}^{\gamma}$ is the corresponding unpolarized fragmentation function. In order to estimate the effects of fragmentation on the asymmetry $A_{\gamma}^{i f}$, we therefore use the LO asymptotic solution for the $\Delta D_{c}^{\gamma}$, which can be derived rather easily. A simple parametrization for the LO asymptotic solution for $N_{F}=4$ active flavors, inspired by the corresponding unpolarized one by Owens [22], is given by

$$
\begin{aligned}
& x \Delta D_{q}^{\gamma}\left(z, Q^{2}\right)=\frac{\alpha}{2 \pi} \ln \frac{Q^{2}}{\Lambda^{2}}\left[e_{q}^{2} \frac{1.52+0.68 z-0.34 z^{2}}{1-1.25 \ln (1-z)} z^{0.7}+0.018(1-z)^{4.56} z^{0.24}\right] \\
& x \Delta D_{g}^{\gamma}\left(z, Q^{2}\right)=\frac{\alpha}{2 \pi} \ln \frac{Q^{2}}{\Lambda^{2}} 0.09 z^{0.63}(1-z)^{1.35}(1-0.59 z) .
\end{aligned}
$$

where $e_{q}$ is the quark's fractional charge and we will set $\Lambda=0.2 \mathrm{GeV}$ in the following. This parametrization is accurate to better than $1 \%$ for $z \geq 0.02$. Fig. 2 shows our results for $z \Delta D_{u}^{\gamma}\left(z, Q^{2}\right) /\left[\alpha \ln \left(Q^{2} / \Lambda^{2}\right) / 2 \pi\right], z \Delta D_{d}^{\gamma}\left(z, Q^{2}\right) /\left[\alpha \ln \left(Q^{2} / \Lambda^{2}\right) / 2 \pi\right]$ and $z \Delta D_{g}^{\gamma}\left(z, Q^{2}\right) /\left[\alpha \ln \left(Q^{2} / \Lambda^{2}\right) / 2 \pi\right]$ vs. $z$ (solid lines), as compared to the Owens parametrization for the unpolarized fragmentation functions [22] (dashed lines). For the quarks we find saturation at large $z$, i.e. $\Delta D_{q}^{\gamma}\left(z, Q^{2}\right) \rightarrow D_{q}^{\gamma}\left(z, Q^{2}\right)$ for $z \rightarrow 1$.

\section{$4 \quad$ Numerical results}

Being equipped with our results for the NLO corrections and the LO fragmentation functions we can now evaluate the effects of these on the asymmetry $A_{\gamma}^{i f}$. It should be noted that a complete NLO analysis of our results is for two reasons not feasible presently. Firstly, as mentioned before, the polarized two-loop anomalous dimensions, needed for a consistent NLO evolution of the polarized parton distributions and fragmentation functions, have not yet been calculated. We therefore have to resort to the LO evolution of the 
polarized parton distributions. Secondly, a consistent NLO prediction would also require knowledge of the fragmentation contribution in NLO, i.e. of the polarized $2 \rightarrow 3$ cross sections $\vec{a} b \rightarrow \vec{c} d e$ (particle $\vec{c}$ fragmenting into the polarized photon), which are unknown. We therefore should not combine our LO expressions for the fragmentation contribution with our NLO corrections to the 'direct' (non-fragmenting) contributions calculated in section 2. Rather, we will first only study the size of the NLO corrections compared to the LO expectations for the 'direct' contributions, in order to check the perturbative stability of the process $\vec{A} B \rightarrow \vec{\gamma} X$. Afterwards, we will combine the LO fragmentation contribution with the LO 'direct' contributions to study the influence of fragmentation on the asymmetry and on the sensitivity to $\Delta G$. Note that the latter calculation will be a complete and fully consistent LO calculation.

We perform our calculations for $\vec{p} p$ collisions at $\sqrt{S}=40 \mathrm{GeV}$, which should be typical of a fixed target experiment with a high energy proton beam. We choose the polarized parton distributions proposed in ref. [23] (and also used in our previous study for the process $\vec{p} \vec{p} \rightarrow \gamma X[8]$ ), where a set of distributions for either of the extreme spin scenarios mentioned in the introduction is given. In both cases the polarized valence quark densities are assumed to be related to the unpolarized ones via

$$
\begin{aligned}
\Delta u_{v}\left(x, Q_{0}^{2}\right) & =x^{0.287} u_{v}\left(x, Q_{0}^{2}\right) \\
\Delta d_{v}\left(x, Q_{0}^{2}\right) & =\left(\frac{x-x_{0}}{1-x_{0}}\right) x^{0.76} d_{v}\left(x, Q_{0}^{2}\right)
\end{aligned}
$$

where $x_{0}=0.75$ and $Q_{0}^{2}=10 \mathrm{GeV}^{2}$. For the spin scenario with a large gluon polarization (hereafter referred to as set $a$ ) the simple parametrization [23]

$$
\Delta G\left(x, Q_{0}^{2}\right)=6 x^{0.76}(1-x)^{3} G\left(x, Q_{0}^{2}\right)
$$

is chosen, together with an $\mathrm{SU}(3)_{\mathbf{f}}$ symmetric sea quark distribution which is assumed to vanish at the input scale $Q_{0}^{2}=10 \mathrm{GeV}^{2}$. In the other extreme scenario (set $b$ ) the gluon distribution $\Delta G\left(x, Q_{0}^{2}\right)$ vanishes, but the $\mathrm{SU}(3)_{\mathrm{f}}$ symmetric polarized sea is related to the unpolarized strange distribution via [23]

$$
\Delta s\left(x, Q_{0}^{2}\right)=-11.8 x^{0.94}(1-x)^{5} s\left(x, Q_{0}^{2}\right)
$$

The above forms chosen for the polarized parton distributions in all cases automatically guarantee that these satisfy the constraints $\left|\Delta f\left(x, Q^{2}\right)\right| \leq f\left(x, Q^{2}\right)$ for all $Q^{2} \geq Q_{0}^{2}$, irrespective of the unpolarized parton distributions chosen. Our choice for the latter in eqs. (31)-(34) is the (leading order) set of Glück, Reya and Vogt [24]. With this choice 
both above sets give a very good description of the EMC data on $g_{1}^{p}\left(x, Q_{0}^{2}\right)[1,25]$. For the unpolarized distributions, needed in the expressions for the polarized as well as the unpolarized cross sections, we also use those of ref. [24]. Apart from being in good agreement with recent HERA data on $F_{2}\left(x, Q^{2}\right)$ [26], these have the advantage that they provide a set of leading order as well as of next-to-leading order structure functions. This allows for a consistent evaluation of the unpolarized cross section in leading (using the leading order set) and in next-to-leading order (using, for consistency, the two-loop evolved set). According to the above discussion we have to stick to the leading order evolution for the polarized parton densities. We work with $N_{F}=4$ flavors, neglecting, however, the contributions from incoming polarized charm quarks. We use the $\Lambda_{\overline{\mathrm{MS}}}$ values and the (one-loop (for LO)/two-loop (for NLO)) expressions for $\alpha_{s}$ according to our choice of the unpolarized structure functions [24]. We assume all scales to be equal and set $\mu=M=M^{\prime}=p_{T}$.

As was shown in the LO studies $[9,10]$, it is interesting to consider rapidity distributions in order to study the sensitivity of $\vec{p} p \rightarrow \vec{\gamma} X$ to $\Delta G$. The reason for this is that the region of negative c.m.s. rapidities $\eta$ (with respect to the direction of the incoming polarized proton) is in LO dominated by $q \Delta G$ contributions, whereas the region of positive $\eta$ is determined by the combination $G \Delta q[9,10]$. This means that the cross sections and asymmetries at negative $\eta$ show the strongest sensitivity to $\Delta G$. Fig. 3 shows our results for the 'direct' unpolarized and polarized cross sections as a function of $\eta$ at $p_{T}=5 \mathrm{GeV}$. It becomes clear that indeed the polarized cross sections for sets $a$ and $b$ differ very much at negative $\eta$, but almost agree at positive $\eta$, which is a result of the polarized valence quark distributions being the same for both sets. We show in Fig. 3 the LO (dashed lines) as well as the NLO (solid lines) results. Obviously, including the NLO corrections does not influence the strong sensitivity to $\Delta G$ in the region $\eta<0$, and the cross sections seem to be rather stable with respect to these corrections, although the latter are not negligible. This can be seen more clearly in Fig. 4 where we show the $K$-factors corresponding to Fig. 3, which are defined by

$$
K \equiv \frac{E_{\gamma} d^{3}(\delta) \sigma^{N L O} / d^{3} p_{\gamma}}{E_{\gamma} d^{3}(\delta) \sigma^{L O} / d^{3} p_{\gamma}}
$$

The $K$-factors are reasonably close to unity. Note the peculiar behavior of the $K$-factor for set $b$ at negative rapidities, which is a consequence of rather large negative contributions from $\vec{q} q$ scattering which are only present in NLO [27]. These contributions are also present for set $a$, but here they are masked by the much larger contribution from $\vec{g} q$ scattering as we will show below. 
Fig. 5 shows the asymmetries $A_{\gamma}^{i f}$ in LO (dashed lines) and NLO (solid lines) corresponding to the cross sections in Fig. 3. Fig. 5 shows the sensitivity of $\vec{p} p \rightarrow \vec{\gamma} X$ to $\Delta G$ at negative $\eta$ most strongly. It can also be seen that the asymmetry seems to be less subject to NLO corrections than the individual cross sections. Such a feature was also found for the NLO corrections to $\vec{p} \vec{p} \rightarrow \gamma X[8]$.

To put more emphasis on the sensitivity to $\Delta G$ we show in Fig. 6 the contributions of the various NLO scattering processes to $A_{\gamma}^{i f}$ for set $a$. The dash-dotted lines in Fig. 6 show the contributions from $\vec{q} g, \vec{g} q, \vec{q} \vec{q}, \vec{q}{ }^{\left({ }^{\prime}\right)}$ and $\vec{g} g$ scattering, respectively, always divided by the full NLO unpolarized cross section. In this way the sum of all dash-dotted curves yields the full NLO asymmetry (solid line), already shown in Fig. 5. For comparison we also show again the LO asymmetry (dashed line) in Fig. 6. As can be seen from Fig. 6 , the $\vec{g} q$ contribution is responsible for more than $90 \%$ of the total signal in the region $\eta<-0.25 . \vec{q} g$ is important only at positive $\eta$ where also contributions from $\vec{q} \vec{q}$ and $\vec{q} q^{\left({ }^{\prime}\right)}$ scattering are present. The contribution from $\vec{g} g$ is so small that it can hardly be seen in Fig. 6 .

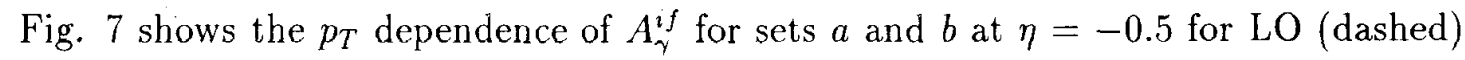
and NLO (solid). The sensitivity to the polarized gluon distribution of the proton also shows up strongly here.

Let us now study the effect of the LO fragmentation contribution. To estimate this contribution we use the polarized fragmentation functions presented in section 3 , i.e. the LO asymptotic solution, along with the corresponding parametrization for the unpolarized case by Owens [22]. For the 'direct' (non-fragmenting) contributions we use our LO results from the previous figures, which makes the results in Figs. 8-10 a complete and consistent LO prediction. Fig. 8 shows the expected cross sections for the unpolarized and the polarized cases. We show the direct contribution (dashed) and the total result (direct+fragmentation, solid) individually. As can be seen, the influence of fragmentation on the polarized cross sections is rather small at negative rapidities, but increases with increasing rapidity. The unpolarized cross section is uniformly increased over the full $\eta$-range. Fig. 9 shows the asymmetry $A_{\gamma}^{i f}$ corresponding to Fig. 8 . Obviously the asymmetry is slightly decreased at negative rapidities, but still allows for a clear distinction between the sets $a$ and $b$. Finally, Fig. 10 shows our results for the $p_{T}$-dependence of $A_{\gamma}^{i f}$ at $\eta=-0.5$. Including the fragmentation contribution again does not sizeably change the asymmetry and thus maintains its strong sensitivity to $\Delta G$. 


\section{Conclusions}

We have calculated the complete NLO $\left(\mathcal{O}\left(\alpha \alpha_{s}^{2}\right)\right)$ corrections to the production of circularly polarized prompt photons in collisions of longitudinally polarized hadrons with unpolarized hadrons. We have found that the cross sections turn out to be perturbatively stable, i.e. the next-to-leading order corrections to them are sizeable but not unreasonably large. We have also found that the polarized cross section and the cross section asymmetry are strongly dependent on the size of the polarized gluon distribution $\Delta G$. This sensitivity to $\Delta G$ shows up in rapidity distributions at $\eta<0$ as well as in $p_{T}$ distributions. We have also examined the effect of including the LO fragmentation contribution. Since no polarized fragmentation functions have been available so far, we have used the asymptotic solution for our calculations, for which we have given a simple parametrization. It turned out that the asymmetries are slightly but not substantially changed by the fragmentation contribution. In this way the process $\vec{p} p \rightarrow \vec{\gamma} X$ appears in leading as well as in nextto-leading order as a promising and reliable tool to decide between presently considered proton spin scenarios, and, if feasible experimentally, it might be a viable alternative to prompt photon experiments with two incoming polarized hadrons.

\section{Acknowledgements}

This work has been supported in part by the 'Bundesministerium für Forschung und Technologie', Bonn. 


\section{Appendix A: $2 \rightarrow 2$ helicity transfer cross sections}

Here we first present the polarized cross sections $d \delta \hat{\sigma}^{\vec{a} b \rightarrow \vec{c} d} / d v(\hat{s}, v, \epsilon)(\vec{c}=\vec{\gamma}$ or $\vec{q})$ as calculated in the HVBM scheme in $n=4-2 \epsilon$ dimensions, needed for the factorization of mass singularities in the next-to-leading order calculation of $\vec{A} B \rightarrow \vec{\gamma} X$. Defining the common factor

$$
\mathcal{N} \equiv \frac{\pi}{\hat{s}} \frac{\mu^{2 \epsilon}}{\Gamma(1-\epsilon)}\left(\frac{4 \pi \mu^{2}}{\hat{s} v(1-v)}\right)^{\epsilon}
$$

we have, using $\hat{t} \equiv\left(p_{a}-p_{c}\right)^{2}=-\hat{s}(1-v)$ and $\hat{u} \equiv\left(p_{b}-p_{c}\right)^{2}=-\hat{s} v$ :

$$
\begin{aligned}
& \frac{d \delta \hat{\sigma}^{\vec{q} q \rightarrow \vec{\gamma} g}}{d v}(\hat{s}, v, \epsilon)=\frac{2 C_{F}}{N_{C}} \alpha \alpha_{s} e_{q}^{2} \mathcal{N}\left[\frac{\hat{u}^{2}-\hat{t}^{2}}{\hat{t} \hat{u}}-\epsilon \frac{\hat{s}^{2}}{\hat{t} \hat{u}}\right] \\
& \frac{d \delta \hat{\sigma}^{\overrightarrow{q g} \rightarrow \vec{\gamma} q}}{d v}(\hat{s}, v, \epsilon)=\frac{1}{N_{C}} \alpha \alpha_{s} e_{q}^{2} \mathcal{N}\left[-\frac{\hat{s}^{2}-\hat{t}^{2}}{\hat{s} \hat{t}}+\epsilon \frac{\hat{u}^{2}}{\hat{s} \hat{t}}\right] \\
& \frac{d \delta \hat{\sigma}^{\vec{g} q \rightarrow \vec{\gamma} q}}{d v}(\hat{s}, v, \epsilon)=\frac{1}{N_{C}} \alpha \alpha_{s} e_{q}^{2} \mathcal{N}\left[-\frac{\hat{s}^{2}+\hat{u}^{2}}{\hat{s} \hat{u}}\right] \\
& \frac{d \delta \hat{\sigma}^{\vec{q} q \rightarrow \vec{q}^{\prime} \vec{q}^{\prime}}}{d v}(\hat{s}, v, \epsilon)=\frac{d \delta \hat{\sigma}^{\hat{q q} \rightarrow \vec{q}^{\prime} q^{\prime}}}{d v}(\hat{s}, v, \epsilon)=\frac{C_{F}}{N_{C}} \alpha_{s}^{2} \mathcal{N}\left[\frac{\hat{u}^{2}-\hat{t}^{2}}{\hat{s}^{2}}\right] \\
& \frac{d \delta \hat{\sigma}^{\vec{g} g \rightarrow \overrightarrow{q q}}}{d v}(\hat{s}, v, \epsilon)=\frac{d \delta \hat{\sigma}^{\vec{g} g \rightarrow \vec{q} q}}{d v}(\hat{s}, v, \epsilon) \\
& =\frac{1}{2 C_{F} N_{C}} \alpha_{s}^{2} \mathcal{N}\left(\hat{u}^{2}-\hat{t}^{2}-\epsilon \hat{s}^{2}\right)\left[\frac{C_{F}}{\hat{t} \hat{u}}-\frac{N_{C}}{\hat{s}^{2}}\right] \\
& \frac{d \delta \hat{\sigma}^{\hat{q} g \rightarrow \vec{q} g}}{d v}(\hat{s}, v, \epsilon)=\frac{1}{N_{C}} \alpha_{s}^{2} \mathcal{N}\left(\left(\hat{s}^{2}+\hat{u}^{2}\right)(1+\epsilon)^{2}+6 \epsilon \hat{s} \hat{u}+2 \epsilon^{2} \hat{s} \hat{u}\right)\left[\frac{N_{C}}{\hat{t}^{2}}-\frac{C_{F}}{\hat{s} \hat{u}}\right] \\
& \frac{d \delta \hat{\sigma}^{\vec{g} q \rightarrow \vec{q} g}}{d v}(\hat{s}, v, \epsilon)=\frac{1}{N_{C}} \alpha_{s}^{2} \mathcal{N}\left(\hat{s}^{2}-\hat{t}^{2}-\epsilon \hat{u}^{2}\right)\left[\frac{N_{C}}{\hat{u}^{2}}-\frac{C_{F}}{\hat{s} \hat{t}}\right] \\
& \frac{d \delta \hat{\sigma}^{\vec{q}^{\prime} \rightarrow \vec{q} q^{\prime}}}{d v}(\hat{s}, v, \epsilon)=\frac{C_{F}}{N_{C}} \alpha_{s}^{2} \mathcal{N}\left[\frac{\hat{s}^{2}+\hat{u}^{2}}{\hat{t}^{2}}+\epsilon\right] \\
& \frac{d \delta \hat{\sigma}^{\vec{q} q^{\prime} \rightarrow \vec{q}^{*} q}}{d v}(\hat{s}, v, \epsilon)=\frac{C_{F}}{N_{C}} \alpha_{s}^{2} \mathcal{N}\left[\frac{\hat{s}^{2}-\hat{t}^{2}}{\hat{u}^{2}}\right] \\
& \frac{d \delta \hat{\sigma}^{\vec{q} q \rightarrow \vec{q} q}}{d v}(\hat{s}, v, \epsilon)=\frac{C_{F}}{N_{C}} \alpha_{s}^{2} \mathcal{N}\left[\frac{\hat{s}^{2}+\hat{u}^{2}}{\hat{t}^{2}}+\epsilon+\frac{\hat{s}^{2}-\hat{t}^{2}}{\hat{u}^{2}}\right. \\
& \left.-\frac{2}{N_{C}}\left(\frac{\hat{s}^{2}}{\hat{t} \hat{u}}-\epsilon\left(\frac{\hat{s}}{\hat{u}}-\frac{\hat{s}}{\hat{t}}\right)+\epsilon(1+\epsilon)\right)\right] \\
& \frac{d \delta \hat{\sigma}^{\vec{q} q \rightarrow \vec{q} q}}{d v}(\hat{s}, v, \epsilon)=\frac{d \delta \hat{\sigma}^{\vec{q} q \rightarrow \vec{q} q}}{d v}(\hat{s}, v, \epsilon) \\
& =\frac{C_{F}}{N_{C}} \alpha_{s}^{2} \mathcal{N}\left[\frac{\hat{s}^{2}+\hat{u}^{2}}{\hat{t}^{2}}+\epsilon+\frac{\hat{u}^{2}-\hat{t}^{2}}{\hat{s}^{2}}\right.
\end{aligned}
$$




$$
\begin{aligned}
& \left.-\frac{2}{N_{C}}\left(\frac{\hat{u}^{2}}{\hat{\hat{s}} \hat{t}}+\epsilon\left(\frac{\hat{u}}{\hat{t}}-\frac{\hat{u}}{\hat{s}}\right)+\epsilon(1+\epsilon)\right)\right] \\
\frac{d \delta \hat{\sigma}^{\vec{q} q \rightarrow \vec{q} q}}{d v}(\hat{s}, v, \epsilon)= & \frac{d \delta \hat{\sigma}^{\vec{q} q \rightarrow \vec{q} q}}{d v}(\hat{s}, v, \epsilon) \\
& =\frac{C_{F}}{N_{C}} \alpha_{s}^{2} \mathcal{N}\left[\frac{\hat{s}^{2}-\hat{t}^{2}}{\hat{u}^{2}}+\frac{\hat{u}^{2}-\hat{t}^{2}}{\hat{s}^{2}}+\frac{2}{N_{C}}\left((1+\epsilon) \frac{\hat{t}^{2}}{\hat{s} \hat{u}}-\epsilon(3+\epsilon)\right)\right] .
\end{aligned}
$$

In these equations we have averaged unpolarized incoming gluons by $1 / 2$, i.e. an additional factor $1 /(1-\epsilon)$ has to be inserted for the respective cross sections. For the calculation of the LO fragmentation contribution we also need the cross sections with polarized gluons in the final state. These we only need in four dimensions. The results read [28]:

$$
\begin{aligned}
\frac{d \delta \hat{\sigma}^{\vec{q} g \rightarrow \vec{g} q}}{d v}-(\hat{s}, v) & =\frac{1}{N_{C}} \frac{\pi \alpha_{s}^{2}}{\hat{s}}\left(\hat{s}^{2}-\hat{t}^{2}\right)\left[\frac{N_{C}}{\hat{u}^{2}}-\frac{C_{F}}{\hat{\hat{s}} \hat{t}}\right] \\
\frac{d \delta \hat{\sigma}^{\vec{g} q \rightarrow \vec{g} q}}{d v}(\hat{s}, v) & =\frac{1}{N_{C}} \frac{\pi \alpha_{s}^{2}}{\hat{s}}\left(\hat{s}^{2}+\hat{u}^{2}\right)\left[\frac{N_{C}}{\hat{t}^{2}}-\frac{C_{F}}{\hat{s} \hat{u}}\right] \\
\frac{d \delta \hat{\sigma}^{\vec{q} q \rightarrow \vec{g} g}}{d v}(\hat{s}, v) & =\frac{d \delta \hat{\sigma}^{\vec{q} q \rightarrow \vec{g} g}}{d v}(\hat{s}, v) \\
& =\frac{2 C_{F}}{N_{C}} \frac{\pi \alpha_{s}^{2}}{\hat{s}}\left(\hat{t}^{2}-\hat{u}^{2}\right)\left[\frac{C_{F}}{\hat{t} \hat{u}}-\frac{N_{C}}{\hat{s}^{2}}\right] \\
\frac{d \delta \hat{\sigma}^{\vec{g} g \rightarrow \vec{g} g}}{d v}(\hat{s}, v) & =\frac{N_{C}}{2 C_{F}} \frac{\pi \alpha_{s}^{2}}{\hat{s}}\left(\hat{s}^{4}-\hat{t}^{4}+\hat{u}^{4}\right) \frac{\hat{s}^{2}+\hat{t}^{2}+\hat{u}^{2}}{\hat{s}^{2} \hat{t}^{2} \hat{u}^{2}}
\end{aligned}
$$




\section{Appendix B: Coefficients $\delta c_{i}$ for the NLO corrections}

Here we present the $\delta c_{i}(i=a, \ldots, d ; 1, \ldots, 13)$. We use the abbreviations

$$
\begin{aligned}
\delta T_{q \bar{q}} & =v^{2}-(1-v)^{2} \\
\delta T_{q g} & =1-(1-v)^{2} \\
\delta T_{g q} & =1+v^{2} \\
X & =1-v w, \\
Y & =1-v+v w, \\
v_{i} & =i-v(i=1, \ldots, 5), \\
b_{0} & =\frac{11}{6} N_{C}-\frac{1}{3} N_{F},
\end{aligned}
$$

where $N_{F}$ is the number of active flavors. As usual we have $C_{F}=4 / 3$ and $N_{C}=3$. Furthermore we have already set $T_{R}=1 / 2$ wherever it appeared. As discussed in the main text, we have absorbed the $\sim e_{q}^{2}$-contributions from $\vec{q} \vec{q} \rightarrow \vec{\gamma} q^{\prime} \bar{q}^{\prime}$ (together with the corresponding contributions from $\vec{q} q \rightarrow \vec{\gamma} q \bar{q}$ ) into the cross section for $\vec{q} \vec{q} \rightarrow \vec{\gamma} g g$ where they appear as $N_{F}$ contributions in the coefficients $\delta c_{1}, \delta c_{2}$ and $\delta c_{13}$. Therefore the coefficients for $\vec{q} \bar{q} \rightarrow \vec{\gamma} q^{\prime} \bar{q}^{\prime}$ are all proportional to $e_{q}^{\prime 2}$ which we have left explicitly in the formulae for the sake of clarity. In all processes involving only one quark flavor we omit the trivial charge factor $e_{q}^{2}$.

\section{a) $\underline{\vec{q} q} \rightarrow \vec{\gamma} g g$}

$$
\begin{aligned}
\delta c_{a} & =-\frac{C_{F}^{2}}{N_{C}} \delta T_{q \bar{q}}\left(3-2 \ln \frac{v_{1}}{v}\right) \\
\delta c_{b} & =-4 \frac{C_{F}^{2}}{N_{C}} \delta T_{q \bar{q}} \\
\delta c_{c} & =-\frac{C_{F}^{2}}{N_{C}}\left(1-4 v-\frac{v}{X} \delta T_{q \bar{q}}-\frac{v v_{1}}{X^{2}}-w \delta T_{q \bar{q}}\right) \\
\delta c_{d} & =0 \\
\delta c_{1} & =\frac{C_{F}}{N_{C}} \delta T_{q \bar{q}} b_{0}\left(\frac{5}{3}+\ln \frac{\mu^{2}}{\hat{s}}-\ln v\right)+C_{F}\left(\frac{2}{3} \delta T_{q \bar{q}}-\frac{\pi^{2}}{3} \delta T_{q \bar{q}}+\frac{1}{2}\left(1-4 v+v^{2}\right) \ln ^{2} v_{1}\right. \\
& \left.-v v_{1} \ln \frac{v_{1}}{v}+\frac{1}{2} v v_{2} \ln ^{2} v\right)+\frac{C_{F}^{2}}{N_{C}}\left(-7 \delta T_{q \bar{q}}+\pi^{2} \delta T_{q \bar{q}}-2 \delta T_{q \bar{q}} \ln v \ln v_{1}+v(2+v) \ln v_{1}\right. \\
& \left.-\left(1-4 v+v^{2}\right) \ln ^{2} v_{1}+\left(v^{2}-2 v_{1}\right) \ln { }^{2} v-v_{1} v_{3} \ln v\right) \\
\delta c_{2} & =\delta T_{q \bar{q}}\left(-\frac{C_{F}}{N_{C}} b_{0}+2 C_{F} \ln v_{1}-4 \frac{C_{F}^{2}}{N_{C}} \ln \frac{v_{1}}{v}\right) \\
\delta c_{3} & =2 \frac{C_{F}}{N_{C}}\left(4 C_{F}-N_{C}\right) \delta T_{q \bar{q}}
\end{aligned}
$$




$$
\begin{aligned}
\delta c_{4} & =-\delta c_{c} \\
\delta c_{5} & =-\frac{2}{X} \frac{C_{F}^{2}}{N_{C}} Y(Y-2 v w)-\frac{C_{F}}{X}\left(\delta T_{q \bar{q}}+v^{2} v_{1} w(1+w)\right) \\
\delta c_{6} & =\delta c_{5} \\
\delta c_{7} & =C_{F} \delta T_{q \bar{q}} \\
\delta c_{8} & =\delta c_{4}+\frac{C_{F}}{N_{C}} \frac{1}{X}\left(2 C_{F}-N_{C}\right)\left(1-4 v+v^{2}+2 v^{2} w-v^{2} w^{2}\right) \\
\delta c_{9} & =4 \frac{C_{F}^{2}}{N_{C}} v-C_{F} v\left(v_{2}-v w\right) \\
\delta c_{10} & =\frac{C_{F}}{N_{C}}\left(4 C_{F}-N_{C}\right) \delta T_{q \bar{q}} \\
\delta c_{11} & =\delta c_{12}=0 \\
\delta c_{13} & =-C_{F} \frac{(X+v)}{2 X^{2}}(3+w-4 v w)(Y-2 v w)+\frac{C_{F}}{N_{C}} b_{0} v\left(v_{2}-v w\right) \\
& +\frac{C_{F}^{2}}{N_{C}} \frac{1}{X^{2}}(Y-2 v w)(2 v-(2-Y)(1+v) w) \\
& \delta c_{a}=\delta c_{b}=\delta c_{c}=\delta c_{1}=\delta c_{2}=\delta c_{3}=\delta c_{5}=\delta c_{6}=\delta c_{7}=\delta c_{9}=\delta c_{10}=\delta c_{12}=0 \\
& \delta c_{d}=\frac{C_{F}}{N_{C}} e_{q}^{\prime 2} \frac{v^{2} v_{1} w}{Y^{2}}(X-v)(X+v) \\
\delta c_{4} & \left.=\delta c_{8}=-\delta c_{d} \quad \mathrm{~b}\right) \\
\delta c_{11} & =-2 \delta c_{d} \\
\delta c_{13} & =\delta c_{d}
\end{aligned}
$$

c) $\underline{\vec{q} g \rightarrow \vec{\gamma} q g}$

$$
\begin{aligned}
\delta c_{a} & =v \delta T_{q g}\left(-3 \frac{C_{F}}{4 N_{C}}-\frac{b_{0}}{2 N_{C}}+\ln \frac{v_{1}}{v}\right) \\
\delta c_{b} & =-\frac{1}{N_{C}}\left(C_{F}+N_{C}\right) v \delta T_{q g} \\
\delta c_{c} & =\frac{C_{F}}{N_{C}} v\left(\frac{-(5-4 v)}{2 X}+\frac{1}{2}\left(2+2 v-v^{2}\right)+\frac{v_{1}}{X^{2}}(3-2 v)-\frac{v_{1}^{2}}{X^{3}}+\frac{w}{2} \delta T_{q g}\right) \\
& +\frac{v^{2}}{v_{1}}\left(-\frac{v_{1}}{v}+\frac{v_{1}^{3}}{X^{3} v}+\frac{v_{1} v_{2}}{X v}(1+v)-\frac{v_{1}^{2} v_{2}}{X^{2} v}+(1-2 v) v_{1} w-v w^{2}(1+v)+v^{2} w^{3}\right) \\
\delta c_{d} & =-\frac{v}{2 Y^{2} v_{1}}(X+v)\left(\frac{C_{F}}{N_{C}} v_{1}^{2}+Y v w\right)\left(v_{1}^{2}+2 Y v w\right) \\
\delta c_{1} & =v \delta T_{q g} \frac{b_{0}}{2 N_{C}} \ln \frac{\mu^{2}}{\hat{s}}+\frac{1}{4 N_{C}}\left(2 C_{F}+N_{C}\right) v \delta T_{q g} \ln ^{2} v+\frac{1}{2} v v_{1} \ln v_{1}+\frac{1}{4} \pi^{2} v v_{1}(1+v) \\
& +\frac{1}{4} v v_{1}(1+v) \ln v_{1} \ln \frac{v_{1}}{v^{2}}+\frac{C_{F}}{N_{C}}\left(-\frac{7}{4} v \delta T_{q g}-\frac{3}{4} v \delta T_{q g} \ln v-\frac{1}{2} v(1-2 v) \ln v_{1} \ln \frac{v_{1}}{v^{2}}\right. \\
& \left.+\frac{1}{2} v(1+2 v) \ln v_{1}-\frac{1}{6} \pi^{2} v\left(3-4 v-v^{2}\right)\right) \\
\delta c_{2} & =v \delta T_{q g}\left(-\ln \frac{v_{1}}{v}-\frac{C_{F}}{4 N_{C}}(3-4 \ln v)\right)
\end{aligned}
$$




$$
\begin{aligned}
& \delta c_{3}=\frac{1}{N_{C}}\left(C_{F}+2 N_{C}\right) v \delta T_{q g} \\
& \delta c_{4}=\frac{C_{F}}{N_{C}} v^{2}\left(\frac{5-4 v}{2 X v}+\frac{2-6 v-3 v^{2}}{2 v}-\frac{v_{1}}{X^{2} v}(3-2 v)+\frac{v_{1}^{2}}{X^{3} v}+\frac{v_{1}^{3}}{Y^{2} v}-\frac{v_{1}^{2} v_{5}}{2 Y v}-\frac{w}{2}(4-11 v)\right) \\
& +\frac{v^{2}}{v_{1}}\left(-\frac{v_{1}^{3}}{X^{3} v}-\frac{v_{1}^{3}}{Y v}-\frac{v_{1} v_{2}}{X v}+\frac{v_{1}^{2} v_{2}}{v}+\frac{v_{1}^{2} v_{2}}{X^{2} v}-\frac{v_{1} v_{3} w}{2}+2 v w^{2}(1+v)-2 v^{2} w^{3}\right) \\
& \delta c_{5}=-\frac{C_{F}}{N_{C}} v^{3}(1+w)-\frac{v}{2 v_{1}}\left((1+v) v_{1}^{2}+2(1-3 v) v v_{1} w-4(2-Y) v^{2} w^{2}\right) \\
& \delta c_{6}=-\frac{C_{F}}{N_{C}} v^{3}(1-2 w)+\frac{v^{2}}{2 X}\left(v_{2}-w-v^{2} w+2 v^{2} w^{2}+v^{2} w^{3}\right) \\
& \delta c_{7}=\frac{1}{2 N_{C}}\left(2 C_{F}-N_{C}\right) v^{2} v_{2} \\
& \delta c_{8}=\delta c_{4}+\frac{C_{F}}{N_{C}} v^{3}(1-2 w)+v^{2}\left(-\frac{v_{2}}{X}-\frac{1}{2} w(2-3 v)+\frac{1}{2} v w^{2}\right) \\
& \delta c_{9}=\frac{C_{F}}{N_{C}} v^{3}(2-w)+\frac{v}{2 v_{1}}\left(\left(1+4 v-3 v^{2}\right) v_{1}+(4-5 v) v v_{1} w-v^{2} w^{2}(5+3 v)+4 v^{3} w^{3}\right) \\
& \delta c_{10}=-\frac{C_{F}}{N_{C}} v(1-2 v)+\frac{v}{2}\left(1+4 v-3 v^{2}\right) \\
& \delta c_{11}=\frac{C_{F}}{N_{C}} v\left(4 v_{1}+\frac{2 v_{1}^{3}}{Y^{2}}-\frac{v_{1}^{2} v_{5}}{Y}-v w(2+v)\right)+v\left(\frac{3-2 v}{2}-\frac{2 v_{1}^{2}}{Y}-\frac{v w}{2}+\frac{v^{2} w^{2}}{2}\right) \\
& \delta c_{12}=\frac{v}{2}(1-2 v)-\frac{C_{F}}{N_{C}} v v_{1}(1+v) \\
& \delta c_{13}=v^{2} w\left(\frac{1}{2}+\frac{4-3 v}{2 X}+\frac{v_{1}}{Y}-\frac{v_{1}}{2 X^{2}}(9-4 v)+\frac{3 v_{1}^{2}}{X^{3}}\right)+\frac{C_{F}}{N_{C}} v^{2}\left(-\frac{1}{4 v}\left(4-6 v+v^{2}\right)\right. \\
& \left.+\frac{v_{1}}{2 X^{2} v}(23-16 v)-\frac{4 v_{1}^{2}}{X^{3} v}+\frac{v_{1}^{2}}{2 Y v}(8+v)-\frac{3 v_{1}^{3}}{2 Y^{2} v}-\frac{v_{3}}{2 X v}(6-7 v)-\frac{v_{2} w}{4}\right) \\
& \text { d) } \underline{\vec{g} q \rightarrow \vec{\gamma} q g} \\
& \delta c_{a}=v_{1} \delta T_{g q}\left(-\frac{b_{0}}{2 N_{C}}-\frac{C_{F}}{4 N_{C}}\left(3-4 \ln \frac{v_{1}}{v}\right)\right) \\
& \delta c_{b}=-\frac{1}{N_{C}}\left(C_{F}+N_{C}\right) v_{1} \delta T_{g q} \\
& \delta c_{c}=-\delta T_{g q} v_{1}(1-2 w)+\frac{C_{F}}{N_{C}}\left(\frac{v v_{1}}{X}-\frac{v_{1}^{2}}{2}(1+2 v)+\frac{w}{2}\left(2-4 v+v^{2} v_{1}\right)-\frac{v^{3} w^{2}}{2}\right) \\
& \delta c_{d}=-\frac{v}{2 Y^{2}}(X+v)(2 Y-v w)\left(v_{1}^{2}+v v_{1} w+\frac{C_{F}}{N_{C}} v^{2} w^{2}\right) \\
& \delta c_{1}=v_{1} \delta T_{g q} \frac{b_{0}}{2 N_{C}} \ln \frac{\mu^{2}}{\hat{s}}+\frac{C_{F}}{N_{C}} v_{1}\left(-\frac{7}{4} \delta T_{g q}+2 v \ln v_{1}+\frac{1}{6} \pi^{2}\left(2-6 v+5 v^{2}\right)+v_{1}^{2} \ln ^{2} v_{1}\right. \\
& \left.+\frac{1}{2}\left(3 v^{2}+2 v_{1}\right) \ln ^{2} v-\frac{1}{4}\left(4 v-3 v_{1}(1+v)\right) \ln v-\left(v^{2}+v_{1}^{2}\right) \ln v \ln v_{1}\right) \\
& +v_{1}\left(-v \ln v_{1}+\frac{v}{2} \ln v+\frac{1}{4} \pi^{2} v v_{2}+\frac{v v_{2}}{4} \ln v \ln \frac{v}{v_{1}^{2}}+\frac{1}{4}\left(3-v_{2}^{2}\right) \ln ^{2} v_{1}\right) \\
& \delta c_{2}=v_{1} \delta T_{g q}\left(-\ln \frac{v_{1}}{v}-\frac{C_{F}}{4 N_{C}}(3-4 \ln v)\right) \\
& \delta c_{3}=\frac{1}{N_{C}}\left(C_{F}+2 N_{C}\right) v_{1} \delta T_{g q}
\end{aligned}
$$




$$
\begin{aligned}
\delta c_{4}= & v_{1}\left(1+2 v^{2}+\frac{v v_{1}}{Y}-\frac{w}{2}\left(4+5 v^{2}\right)\right) \\
& +\frac{C_{F}}{N_{C}}\left(-\frac{v v_{1}}{X}+\frac{v_{1}^{2}}{2}(1+3 v)+\frac{v v_{1}^{3}}{Y^{2}}-\frac{v v_{1}^{2} v_{3}}{2 Y}-w\left(1-2 v-v^{3}\right)\right) \\
\delta c_{5}= & \frac{3 v^{2} v_{1} w}{2}+\frac{C_{F}}{N_{C}} v\left(v_{1} v_{2}-(1-3 v) v w\right) \\
\delta c_{6}= & \frac{v_{1}}{2}\left(7 v^{2}+2 v_{1}-3 v^{2} w+2 v^{2} w^{2}\right)+\frac{C_{F}}{N_{C}} v\left(v_{1}(1-2 v)-v w(Y-2 v w)\right) \\
\delta c_{7}= & \frac{v_{1}}{2 N_{C}}\left(2 C_{F}\left(v^{2}+v_{1}^{2}\right)+N_{C} v v_{2}\right) \\
\delta c_{8}= & \delta c_{4}-\frac{C_{F}}{N_{C}} v\left(v^{2}+v_{1}^{2}-2 v^{2} w+v^{2} w^{2}\right)-\frac{v_{1}}{2}\left(3 v^{2}+2 v_{1}-v^{2} w+2 v^{2} w^{2}\right) \\
\delta c_{9}= & \frac{v v_{1} v_{2}}{2}-\frac{C_{F}}{N_{C}} v\left(v+3 v_{1}^{2}-v w(Y-2 v w)\right) \\
\delta c_{10}= & \frac{C_{F}}{N_{C}} v_{1}\left(2 v+3 v_{1}^{2}\right)+\frac{v_{1}}{2}\left(3-v_{2}^{2}\right) \\
\delta c_{11}= & \frac{v v_{1}}{2 Y}\left(v_{1}(2+v)-v v_{2} w\right)-\frac{C_{F}}{N_{C}} \frac{v v_{1}}{Y^{2}}(X+v)\left(v_{1}^{2}+v w(3 Y-2 v w)\right) \\
\delta c_{12}= & \frac{v_{1}}{2}(1-2 v)-\frac{C_{F}}{N_{C}} v_{1}\left(1+v_{1}^{2}\right) \\
\delta c_{13}= & v_{1}\left(-\frac{v}{X}-\frac{1+v}{2}+\frac{v v_{1}}{Y}+\frac{w}{2}(3+v)\right)+\frac{C_{F}}{N_{C}}\left(\frac{1}{4}\left(2+v-v^{3}\right)+\frac{v v_{1}}{X}\right. \\
& \left.-\frac{3 v v_{1}^{3}}{2 Y^{2}}-\frac{v v_{1}^{3}}{2 Y}-\frac{v_{1} w}{2}\left(v^{2}-3 v_{1}^{2}\right)+\frac{v^{3} w^{2}}{2}\right)
\end{aligned}
$$$$
\begin{aligned}
\delta c_{a} & =\delta c_{b}=\delta c_{1}=\delta c_{2}=\delta c_{3}=\delta c_{7}=\delta c_{10}=\delta c_{12}=0 \\
\delta c_{c} & =\frac{1}{N_{C}} v\left(-\frac{v_{1}}{2}(5-3 v)-\frac{2 v_{1}^{2}}{X^{2}}+\frac{2 v_{1} v_{2}}{X}-v w(3-2 v)-\frac{v^{2} w^{2}}{2}\right) \\
\delta c_{d} & =\frac{v}{2 Y^{2}}(X+v)(Y-2 v w)\left(-\frac{v v_{1} w}{C_{F}}+\frac{1}{N_{C}}\left(v_{1}^{2}+v w(2 Y-v w)\right)\right. \\
\delta c_{4} & =\frac{1}{2 C_{F}} v^{2} v_{1} w\left(5+\frac{4 v_{1}}{Y^{2}}-\frac{4}{Y v_{2}}\left(v^{2}+3 v_{1}\right)-\frac{2}{X v_{2}}\left(1+v_{1}^{2}\right)\right) \\
& +\frac{2 v}{N_{C}}\left(-v_{1}(1+v)+\frac{v_{1}^{2}}{X^{2}}-\frac{2 v_{1}^{2}}{X v_{2}}+\frac{v_{1}}{Y v_{2}}\left(1+v_{1}^{2}\right)+v v_{2} w\right) \\
\delta c_{5} & =\frac{1}{2 C_{F}} v v_{1} w\left(-(2+v)+\frac{2}{X v_{2}}\left(1+v_{1}^{2}\right)+\frac{2}{Y v_{2}}\left(1+v_{1}^{2}\right)\right) \\
& +\frac{1}{N_{C}} v\left(v_{1}^{2}-\frac{2 v_{1}}{X v v_{2}}\left(1+v_{1}^{2}\right)+\frac{2 v_{1}^{2}}{Y v v_{2}}\left(1+v_{1}^{2}\right)+2 w\right) \\
\delta c_{6} & =\frac{1}{C_{F}} \frac{v^{2} v_{1} w}{2}\left(1-\frac{2}{Y v v_{2}}\left(1+v_{1}^{2}\right)+\frac{2 v_{1}}{X v v_{2}}\left(1+v_{1}^{2}\right)-2 w\right) \\
& +\frac{1}{N_{C}}\left(4 v_{1}^{2}-\frac{2 v_{1}^{2}}{X Y}\left(1+v_{1}^{2}\right)+v^{2} v_{2} w^{2}\right) \\
\delta c_{8} & =\frac{1}{C_{F}} v^{2} v_{1} w\left(\frac{2 v_{1}}{Y^{2}}-\frac{1}{Y v v_{2}}\left(-2+8 v-7 v^{2}+2 v^{3}\right)-\frac{1+v_{1}^{2}}{X v v_{2}}+w\right)
\end{aligned}
$$ 


$$
\begin{aligned}
& +\frac{1}{N_{C}}\left(-4+\frac{2 v v_{1}^{2}}{X^{2}}+3 v\left(1+v_{1}^{2}\right)+\frac{2 v_{1}}{Y v_{2}}\left(1+v_{1}^{2}\right)-\frac{2 v_{1}^{2}}{X v_{2}}\left(2-v_{2}^{2}\right)+4 v^{2} v_{2} w-v^{2} v_{2} w^{2}\right) \\
\delta c_{9} & =-\frac{v}{N_{C}}\left(v^{2}+v_{1}^{2}+v w(2 Y-v w)\right) \\
\delta c_{11} & =\frac{1}{C_{F}} \frac{v^{2} v_{1} w}{Y^{2}}(X+v)(Y-2 v w) \\
\delta c_{13}=\frac{1}{C_{F}} v^{2} v_{1} w\left(-2-\frac{2 v_{1}}{X^{2}}-\frac{4 v_{1}}{Y^{2}}+\frac{v_{2}}{X}+\frac{2 v_{2}}{Y}\right)+\frac{1}{N_{C}} \frac{v^{2} v_{1}}{X^{2}}(1-w)\left(1+v^{2} w^{2}\right) & \text { f) } \underline{\vec{q} q \rightarrow \vec{\gamma} q q}
\end{aligned}
$$

$$
\begin{aligned}
\delta c_{a} & =\delta c_{b}=\delta c_{1}=\delta c_{2}=\delta c_{3}=\delta c_{7}=\delta c_{10}=\delta c_{12}=0 \\
\delta c_{c} & =\frac{C_{F}}{N_{C}}\left(-1-\frac{v v_{1}}{2 X^{2}}-v^{2} v_{1}+\frac{v v_{3}}{2 X}-\frac{w}{2}\left(2 v^{3}-v_{1}\right)-\frac{v^{3} w^{2}}{v_{1}}(1+v)+\frac{v^{4} w^{3}}{v_{1}}\right) \\
\delta c_{d} & =-\frac{v}{v_{1}}(X+v)\left(-\frac{C_{F}}{N_{C}^{2}} v_{1}\left(v_{1}+v w\right)+\frac{C_{F}}{N_{C}}\left(v_{1}^{2}+v^{2} w^{2}\right)\right) \\
\delta c_{4} & =-\frac{C_{F}}{N_{C}^{2}} \frac{v}{X}(X+v)\left(v_{1}+v w(3-Y)\right)+\frac{C_{F}}{N_{C}} \frac{1}{v_{1}}\left(\frac{v v_{1}^{2}}{2 X^{2}}+v_{1}\left(7 v-2 v^{3}+v_{1}(1+v)\right)\right. \\
& \left.-\frac{v v_{1} v_{3}}{2 X}-\frac{2 v v_{1}^{2} v_{3}}{Y}-\frac{v_{1} w}{2}\left(6 v^{2}-4 v^{3}+v_{1}\right)+2 v^{3}(1+v) w^{2}-2 v^{4} w^{3}\right) \\
\delta c_{5} & =\frac{C_{F}}{N_{C}} \frac{v^{2} w}{v_{1}}\left(v_{1}(1+v)-\frac{2 v_{1}^{2}}{Y}+2 v w(1+v)-2 v^{2} w^{2}\right)+\frac{C_{F}}{N_{C}^{2}} \frac{(X+v)}{X}\left(-v_{1} X(1+v)+v^{3} w^{2}\right) \\
\delta c_{6} & =\frac{C_{F}}{N_{C}} \frac{v^{2} v_{1}}{Y}\left(2 v_{1}+X w+3 v w\right)+\frac{C_{F}}{N_{C}^{2}} \frac{(X+v)}{X}\left(X-v v_{2}-v^{3} w(1-w)\right) \\
\delta c_{8} & =\delta c_{4}+\frac{C_{F}}{N_{C}} \frac{v}{Y}\left(v_{1}(1+v)+v w(5-3 X-4 Y)\right)-\frac{C_{F}}{N_{C}^{2}} \frac{(X+v)}{X}\left(X-v v_{2}-v^{3} w(1-w)\right) \\
\delta c_{9} & =\frac{C_{F}}{N_{C}} \frac{v}{v_{1}}\left(v_{1}(1+v)-v v_{1} w(2+v)-2 v^{2} w^{2}(2-Y)\right) \\
\delta c_{11} & =2 \frac{C_{F}}{N_{C}} v(X-v) \\
\delta c_{13} & =\frac{C_{F}}{N_{C}}\left(\frac{v}{2 X}(5-4 v)-\frac{v v_{1}}{2 X^{2}}-\frac{1}{2}\left(1+4 v v_{1}\right)+\frac{w}{2}\left(v^{3}+v_{1}(2-3 v)\right)\right)
\end{aligned}
$$

g) $\underline{q} \vec{q} \rightarrow \vec{\gamma} q \bar{q}$

$$
\begin{aligned}
\delta c_{a} & =\delta c_{b}=\delta c_{1}=\delta c_{2}=\delta c_{3}=0 \\
\delta c_{c} & =\frac{C_{F}}{N_{C}}\left(-1-\frac{v v_{1}}{2 X^{2}}-v^{2} v_{1}+\frac{v v_{3}}{2 X}-\frac{w}{2}\left(2 v^{3}-v_{1}\right)-\frac{v^{3} w^{2}}{v_{1}}(1+v)+\frac{v^{4} w^{3}}{v_{1}}\right) \\
\delta c_{d} & =\frac{C_{F}}{N_{C}^{2}} \frac{v}{Y^{2}}(X+v)(Y-2 v w)\left(v_{1}^{2}+Y v w\right)+\frac{C_{F}}{N_{C}} \frac{v}{v_{1}}(2-Y)\left(-2 v_{1}^{2}+\frac{3 v_{1}^{3}}{Y}-\frac{2 v_{1}^{4}}{Y^{2}}-v^{2} w^{2}\right) \\
\delta c_{4} & =\frac{C_{F}}{N_{C}^{2}} v\left(v_{1}^{2}+\frac{2 v_{1}^{2}}{Y}-\frac{4 v_{1}^{3}}{Y^{2}}-v w(1-3 v)-v^{2} w^{2}\right)+\frac{C_{F}}{N_{C}}\left(-\left(3 v^{2}-v_{1}\right)+\frac{v v_{1}}{2 X^{2}}\right. \\
& \left.-\frac{2 v v_{1}}{Y}\left(1-4 v+v^{2}\right)+\frac{4 v v_{1}^{3}}{Y^{2}}-\frac{v v_{3}}{2 X}+\frac{w}{2}\left(6 v^{3}-v_{1}\right)+\frac{w^{2}}{v_{1}} 2 v^{3}(1+v)-\frac{2 v^{4} w^{3}}{v_{1}}\right) \\
\delta c_{5} & =\frac{C_{F}}{N_{C}} \frac{v^{2} w}{v_{1}}\left(v_{1}(1+v)+\frac{2 v_{1}^{2}}{Y}+2 v w(1+v)-2 v^{2} w^{2}\right)
\end{aligned}
$$




$$
\begin{array}{rl} 
& +\frac{C_{F}}{N_{C}^{2}} v\left(-(2-3 v)+\frac{4 v_{1}^{2}}{Y}+v w(3-2 v)-v^{2} w^{2}\right) \\
\delta c_{6} & =-\frac{C_{F}}{N_{C}} \frac{v^{2} v_{1}}{Y}\left(-2 v_{1}+3 v_{1} w+v w^{2}\right)-\frac{C_{F}}{N_{C}^{2}} \frac{v v_{1}}{Y}\left(X-v^{2}-v^{2} w+2 v^{2} w^{2}\right) \\
\delta c_{7} & =\frac{C_{F}}{N_{C}^{2}} v_{1}(1+v) \\
\delta c_{8} & =\delta c_{4}-\frac{C_{F}}{N_{C}^{2}} \frac{v^{2} v_{1} w}{Y}(1+3 v-3 v w)+\frac{C_{F}}{N_{C}} \frac{v}{Y}\left(-v_{1}(1+v)+2 v w-4 v^{2} w+v^{2} w^{2}\right) \\
\delta c_{9} & =\frac{C_{F}}{N_{C}^{2}} v\left(-2+X v+v^{2} w^{2}\right)-\frac{C_{F}}{N_{C}} \frac{v}{v_{1}}\left(v_{1}(1+v)+v^{2} v_{1} w+2 v^{2} w^{2}(2-Y)\right) \\
\delta c_{10} & =-\frac{C_{F}}{N_{C}^{2}} v v_{2} \\
\delta c_{11} & =\frac{C_{F}}{N_{C}^{2}} v\left(-\left(11-13 v+3 v^{2}\right)-\frac{8 v_{1}^{3}}{Y^{2}}+\frac{4 v_{1}^{2} v_{4}}{Y}+v w(5-2 v)-v^{2} w^{2}\right) \\
& +\frac{2 C_{F}}{N_{C}} \frac{v}{Y^{2}}(X-v)\left(v_{1}^{2}+v w v_{1}^{2}+v^{2} w^{2} v_{2}\right) \\
\delta c_{12}= & -\frac{C_{F}}{N_{C}^{2}}(1-2 v) \\
\delta c_{13}= & \frac{C_{F}}{N_{C}^{2}}(Y-2 v w)\left(-\frac{1}{2 X v}\left(1-6 v+2 v^{2}\right)+\frac{1}{2 v}\left(1-2 v+4 v^{2}\right)-\frac{v_{1}}{2 X^{2}}\right) \\
& +\frac{C_{F}}{N_{C}}\left(\frac{v}{2 X}-\frac{v v_{1}}{2 X^{2}}-\frac{4 v v_{1}^{3}}{Y^{2}}-\frac{1}{2}\left(1+4 v v_{1} v_{2}\right)+\frac{2 v v_{1}^{2} v_{4}}{Y}+\frac{w}{2}\left(v^{2} v_{1}+v_{2}\right)\right) \\
h & h \vec{q}^{\prime} \rightarrow \vec{\gamma} q q^{\prime}
\end{array}
$$

$$
\begin{aligned}
\delta c_{a} & =\delta c_{b}=\delta c_{1}=\delta c_{2}=\delta c_{3}=\delta c_{7}=\delta c_{10}=\delta c_{12}=0 \\
\delta c_{c} & =-e_{q}^{\prime 2} \frac{C_{F}}{2 N_{C}} v_{1}\left(1+v^{2}\right)(2-w)-e_{q}^{2} \frac{C_{F}}{N_{C}} \frac{v^{3} w^{2}}{2 X^{2} v_{1}}(1+X)\left(1+v^{2}-2 v w(2-Y)\right) \\
\delta c_{d} & =\frac{C_{F}}{N_{C}} \frac{v(X+v)}{2 Y v_{1}}\left(-e_{q}^{\prime 2} v_{1}^{2}(2 Y-v w)-e_{q}^{2} v w\left(v_{1}^{2}+2 Y v w\right)\right) \\
\delta c_{4} & =2 e_{q} e_{q}^{\prime} \frac{C_{F}}{N_{C}} \frac{v^{2} w}{Y}(1+X)+e_{q}^{2} \frac{C_{F}}{N_{C}} v_{1}\left(1+2 v^{2}+\frac{v v_{1}}{Y}-\frac{w}{2}\left(1+2 v^{2}\right)\right) \\
& +e_{q}^{2} \frac{C_{F}}{N_{C}} \frac{v^{2} w}{v_{1}}\left(-\frac{v_{1}}{X}+v v_{1}+\frac{v_{1}^{2}}{2 X^{2}}+\frac{v_{1}^{2}}{Y}+2 v w(1+v)-2 v^{2} w^{2}\right) \\
\delta c_{5} & =-2 e_{q} e_{q}^{\prime} \frac{C_{F}}{N_{C}} \frac{v^{2} v_{1} w}{Y}+e_{q}^{2} \frac{C_{F}}{N_{C}} \frac{v^{2} w}{v_{1}}\left(v_{1}(1+v)+2 v w(2-Y)\right) \\
\delta c_{6} & =e_{q}^{\prime 2} \frac{C_{F}}{N_{C}} v^{2} v_{1}(2-w)+2 e_{q} e_{q}^{\prime} \frac{C_{F}}{N_{C}} \frac{v^{2} v_{1} w}{Y} \\
\delta c_{8} & =\delta c_{4}+e_{q} e_{q}^{\prime} \frac{C_{F}}{N_{C}} v\left(-(1-3 v)+\frac{2 v_{1}^{2}}{Y}-v w\right) \\
\delta c_{9} & =e_{q} e_{q}^{\prime} \frac{C_{F}}{N_{C}} v(X+v)-e_{q}^{2} \frac{C_{F}}{N_{C}} \frac{v^{2} w}{v_{1}}\left(v_{1}(1+v)+2 v w(2-Y)\right) \\
\delta c_{11} & =2 \frac{C_{F}}{N_{C}} \frac{v}{Y}\left(e_{q}^{\prime 2} v_{1}^{2}-e_{q}^{2} v^{2} w^{2}\right) \\
\delta c_{13} & =e_{q}^{2} \frac{C_{F}}{N_{C}} v^{2} w\left(\frac{v}{2}-\frac{v_{1}}{2 X^{2}}+\frac{v_{2}}{2 X}\right)-e_{q} e_{q}^{\prime} \frac{C_{F}}{N_{C}} \frac{v v_{1} w}{X}(Y-2 v w)-e_{q}^{2} \frac{C_{F}}{2 N_{C}} v_{1}\left(1+v-v_{2} w\right)
\end{aligned}
$$




\section{References}

[1] J. Ashman et al., EMC Collab., Phys. Lett. B206, 364 (1988); Nucl. Phys. B328, 1 (1989).

[2] J. Ellis, R.A. Flores and S. Ritz, Phys. Lett. B198, 393 (1987);

J. Ellis and R.A. Flores, Nucl. Phys. B307, 883 (1988);

S.J. Brodsky, J. Ellis and M. Karliner, Phys. Lett. B206, 309 (1988);

J. Ellis and M. Karliner, Phys. Lett. B213, 73 (1988);

M. Glück and E. Reya, Z. Phys. C39, 569 (1988).

[3] G. Altarelli and G.G. Ross, Phys. Lett. B212, 391 (1988);

A.V. Efremov and O.V. Teryaev, Dubna report E2-88-287 (1988), published in the proceedings of the Int. Hadron Symposium, Bechyne, Czechoslowakia, 1988, eds. X. Fischer et al. (Czech. Academy of Science, Prague, 1989), p. 302;

G. Altarelli and W.J. Stirling, Particle World 1, 40 (1989).

[4] E.L. Berger and J. Qiu, Phys. Rev. D40, 778 (1989); D40, 3128 (1989);

S. Gupta, D. Indumathi and M.V.N. Murthy, Z. Phys. C42, 493 (1989); Erratum C44, 356 (1989);

H.Y. Cheng and S.N. Lai, Phys. Rev. D41, 91 (1990);

C. Bourrely, J.Ph. Guillet and J. Soffer, Nucl. Phys. B361, 72 (1991);

P. Chiappetta, P. Colangelo, J.Ph. Guillet and G. Nardulli, Z. Phys. C59, 629 (1993).

[5] P. Mathews and R. Ramachandran, Z. Phys. C53, 305 (1992).

[6] A.P. Contogouris, B. Kamal, Z. Merebashvili and F.V. Tkachov, Phys. Lett. B304, 329 (1993); Phys. Rev. D48, 4092 (1993).

[7] L.E. Gordon and W. Vogelsang, Phys. Rev. D48, 3136 (1993).

[8] L.E. Gordon and W. Vogelsang, Phys. Rev. D49, 170 (1994).

[9] N.S. Craigie, K. Hidaka, M. Jacob, A. Penzo and J. Soffer, Nucl. Phys. B204, 365 (1982).

[10] M. Glück and W. Vogelsang, Phys. Lett. B277, 515 (1992). 
[11] RHIC Spin Collab., D. Hill et al., letter of intent RHIC-SPIN-LOI-1991;

G. Bunce et al., Particle World 3, I (1992);

G. Bunce, Conference Proceedings AIP, New York, Particles and Fields Series 42, 147 (1991).

[12] Note that a factor $1 / 2$ is not included in the following definition since one of the polarized particles is outgoing.

[13] Note that we do not consider the fragmentation of an outgoing parton into a polarized photon at this point.

[14] G. 't Hooft and M. Veltman, Nucl. Phys. B44, 189 (1972).

[15] P. Breitenlohner and D. Maison, Commun. math. Phys. 52, 11 (1977).

[16] We have used the program 'Tracer' [17] to calculate the Dirac traces in the HVBM $[14,15]$ scheme.

[17] M. Jamin and M.E. Lautenbacher, 'Tracer' (Version 1.1), TU München report TUMT31-20/91.

[18] R.K. Ellis, M.A. Furman, H.E. Haber and I. Hinchliffe, Nucl. Phys. B173, 397 (1980).

[19] G. Altarelli and G. Parisi, Nucl. Phys. B126, 298 (1977).

[20] Note furthermore that these choices for $\Delta f_{q q}$ and $\Delta f_{g g}$ correspond to the convention commonly adopted in polarized deep-inelastic scattering, since they lead to the following structure of (e.g.) the first moment of the spin-dependent structure function $g_{1}^{P}$ in NLO [21]: $g_{1}^{P}=\frac{1}{2} \sum_{q} e_{q}^{2}\left[\left(1-\frac{\alpha_{s}}{\pi}\right)(\Delta q+\Delta \bar{q})-\frac{\alpha_{s}}{2 \pi} \Delta G\right]$.

[21] W. Vogelsang, Z. Phys. C50, 275 (1991).

[22] J.F. Owens, Rev. Mod. Phys. 59, 465 (1987).

[23] H.-Y. Cheng and C.F. Wai, Phys. Rev. D46, 125 (1992).

[24] M. Glück, E. Reya and A. Vogt, Z. Phys. C53, 127 (1992).

[25] In order to calculate $g_{1}^{p}\left(x, Q_{0}^{2}\right)$ for the scenario with a large $\Delta G$ we have used the gluonic Wilson coefficient $\Delta f_{g}(z)=\frac{1}{2}(2 z-1)\left(\ln \frac{1-z}{z}-1\right)$ by which the gluonic contribution enters $g_{1}^{p}\left(x, Q_{0}^{2}\right)$. This is in accord with the factorization prescription adopted in our calculation of the next-to-leading order corrections (see also [20]). 
[26] I. Abt et al., (H1 Collab.), Nucl. Phys. B407, 515 (1993); M. Derrick et al., (ZEUS Collab.), Phys. Lett. B316, 412 (1993).

[27] Such a sizeable negative contribution from $\vec{q} \vec{q}$ scattering in NLO was also found for the two-spin case $\vec{p} \vec{p} \rightarrow \gamma X$ in refs. $[6,8]$.

[28] R. Gastmans and T.T. Wu, 'The Ubiquitous Photon', Oxford Science Publications (1990).

\section{Figure Captions}

Fig. 1: Representative Feynman diagram for $\vec{q} g \rightarrow \vec{\gamma} q g$ in the situation where the outgoing photon becomes collinear with the outgoing quark, $k_{1}=k_{2} x /(1-x)$. The dotted box frames the $n$-dimensional $2 \rightarrow 2$ process $\vec{q} g \rightarrow \vec{q} g$.

Fig. 2: LO asymptotic solutions for the photon fragmentation functions for up and down quarks and the gluon. Solid lines: Polarized distributions according to the parametrizations in eqs. (29), (30). Dashed lines: Unpolarized distributions according to the Owens parametrization [22].

Fig. 3: Cross sections $E_{\gamma} d^{3}(\delta) \sigma / d^{3} p_{\gamma}$ for the unpolarized and the polarized (sets $a$ and $b$, according to eqs. (31)-(34)) cases vs. c.m.s. rapidity $\eta$ at $\sqrt{S}=40 \mathrm{GeV}$ and $p_{T}=5 \mathrm{GeV}$ in LO (dashed lines) and NLO (solid lines).

Fig. 4: $K$-factors, defined according to eq. (35), corresponding to Fig. 3.

Fig. 5: The asymmetry $A_{\gamma}^{\text {if }}$, defined according to eq. (1), corresponding to Fig. 3. Dashed lines: LO, solid lines: NLO.

Fig. 6: The asymmetry $A_{\gamma}^{i f}$ for set $a$ (as in Fig. 5) in leading (dashed line) and next-toleading (solid line) order, decomposed into the next-to-leading order contributions originating from $\vec{q} g, \vec{g} q, \vec{g} g, \vec{q} \bar{q}$ and $\vec{q} q^{\left({ }^{\prime}\right)}$ scattering as described in the text (dashdotted lines). The dash-dotted curves were obtained by dividing the polarized nextto-leading order cross sections arising from the respective subprocesses by the full next-to-leading order unpolarized cross section, that is, the sum of the dash-dotted lines yields the full next-to-leading order asymmetry (solid curve).

Fig. 7: The asymmetry $A_{\gamma}^{i f}$ as a function of $p_{T}$ at $\sqrt{S}=40 \mathrm{GeV}$ and $\eta=-0.5$. Dashed lines: LO, solid lines: NLO. 
Fig. 8: LO cross sections $E_{\gamma} d^{3}(\delta) \sigma / d^{3} p_{\gamma}$ for the unpolarized and the polarized (sets $a$ and $b$, according to eqs. (31)-(34)) cases vs. c.m.s. rapidity $\eta$ at $\sqrt{S}=40 \mathrm{GeV}$ and $p_{T}=5 \mathrm{GeV}$ in LO. Dashed lines: 'Direct' contributions only, solid lines: Total (i.e. direct+fragmentation) LO result.

Fig. 9: The asymmetry $A_{\gamma}^{i f}$ corresponding to Fig. 8 .

Fig. 10: The asymmetry $A_{\gamma}^{2 f}$ as a function of $p_{T}$ at $\sqrt{S}=40 \mathrm{GeV}$ and $\eta=-0.5$. Dashed lines: 'Direct' contributions only, solid lines: Total (i.e. direct+fragmentation) LO result. 


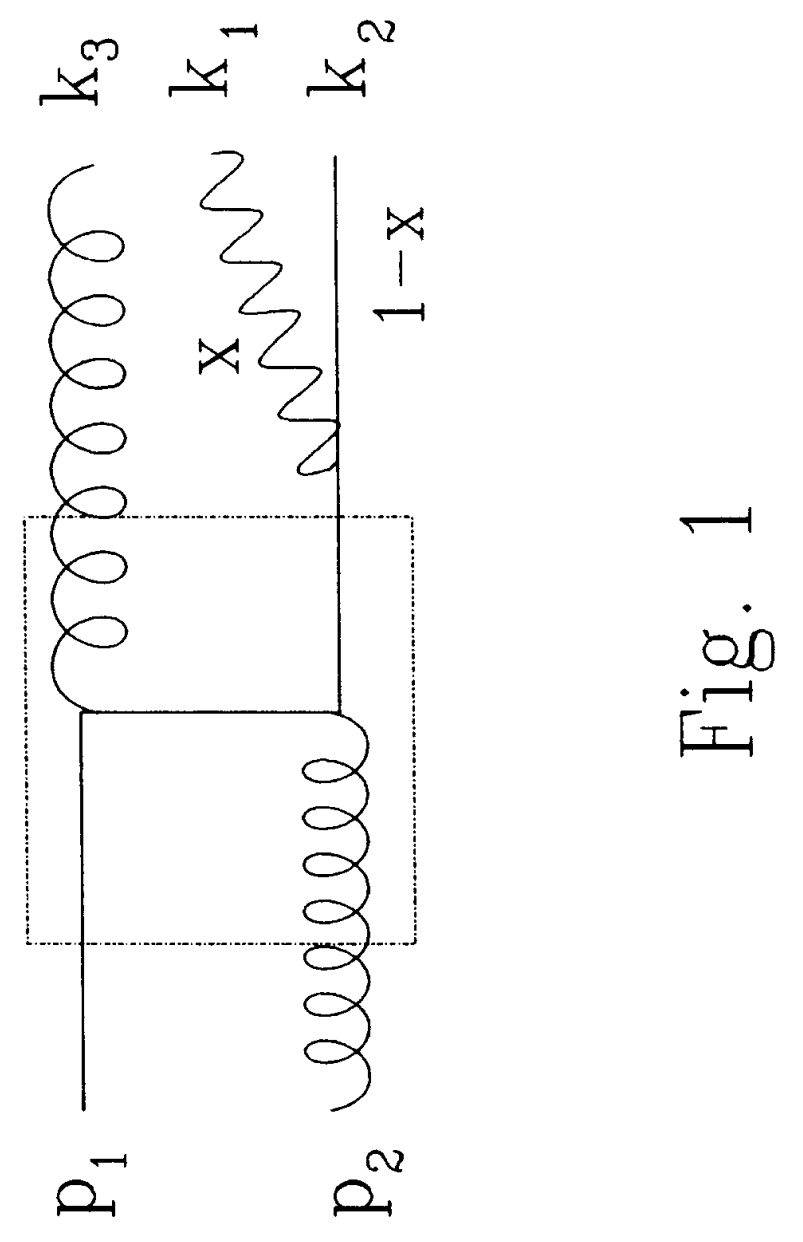




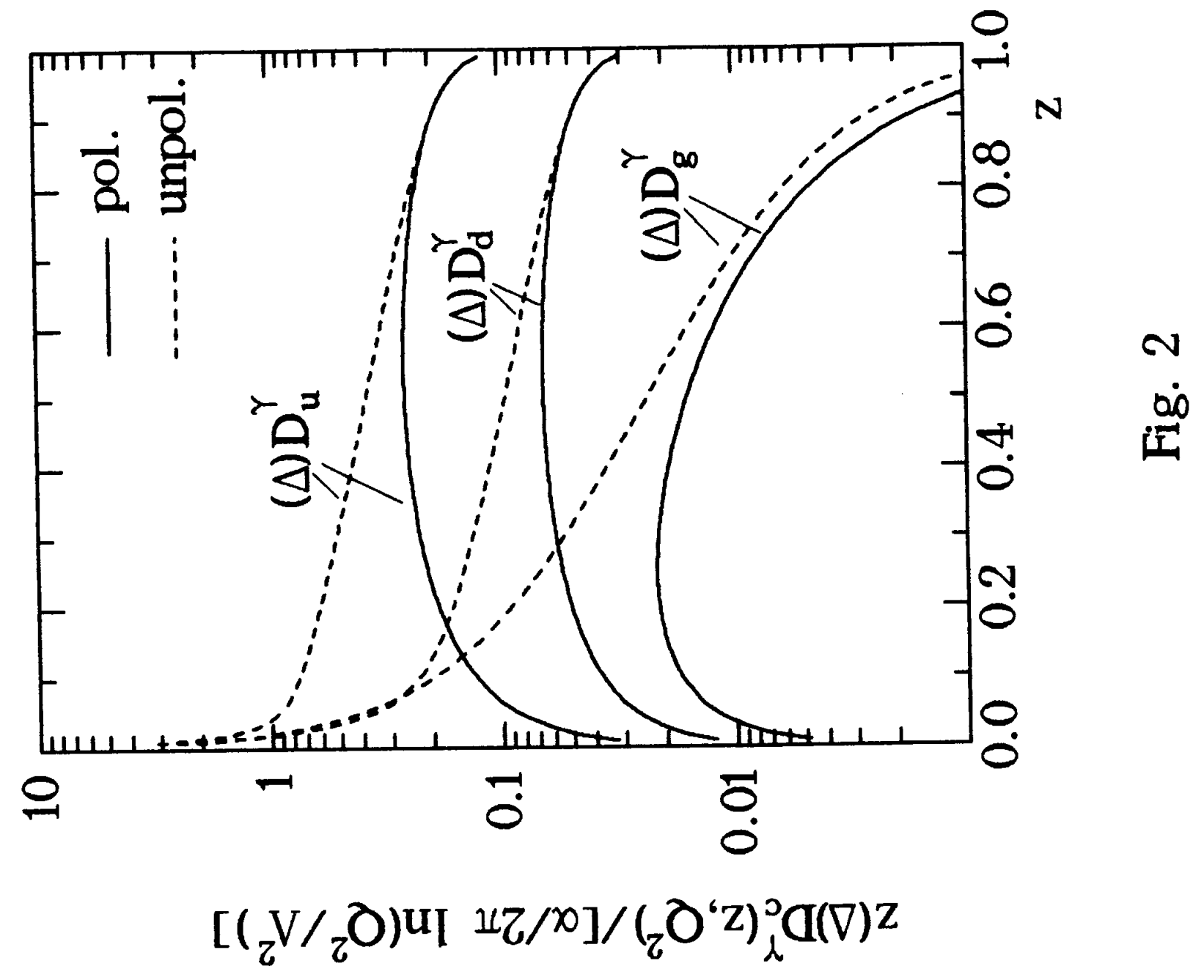




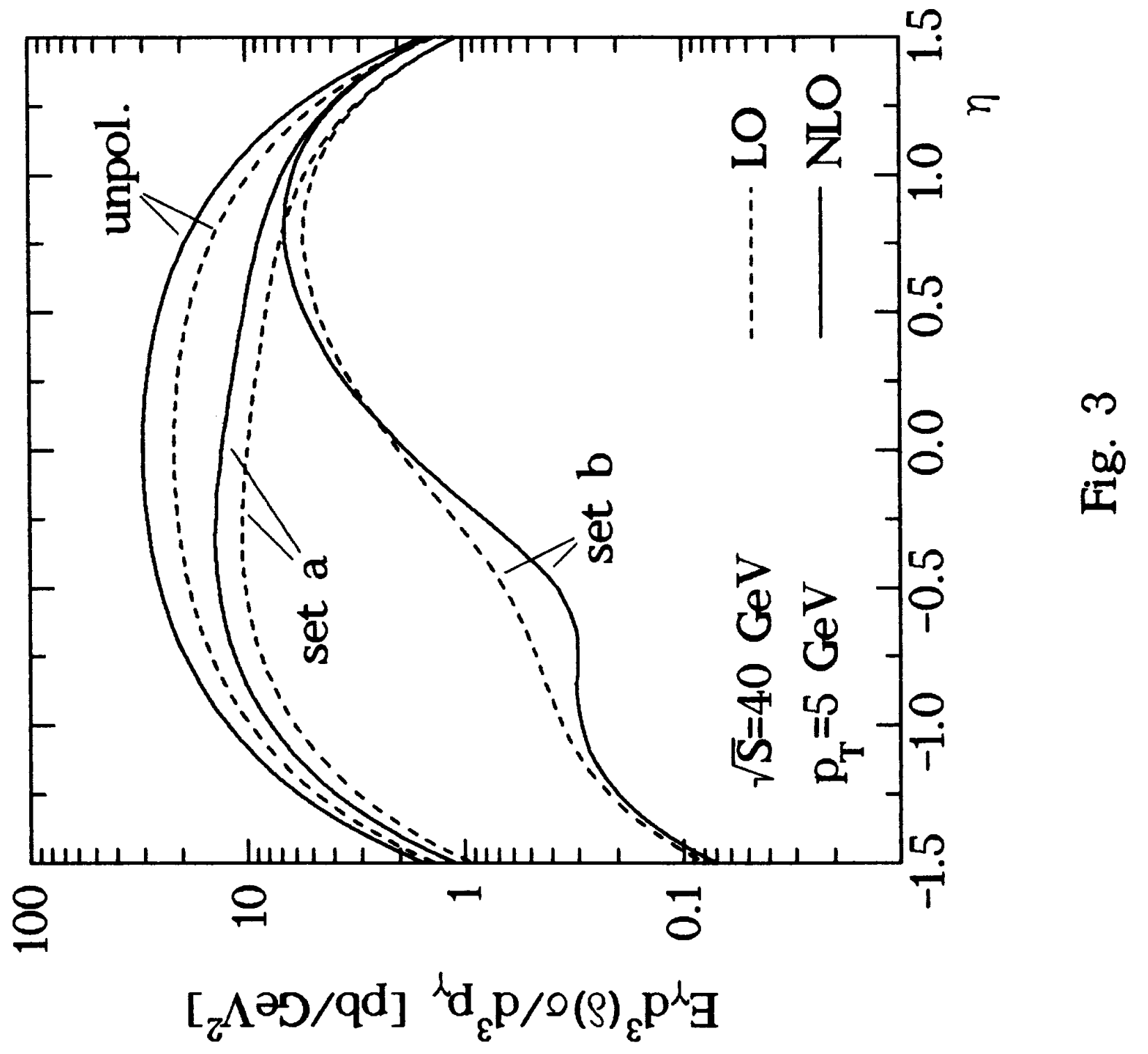




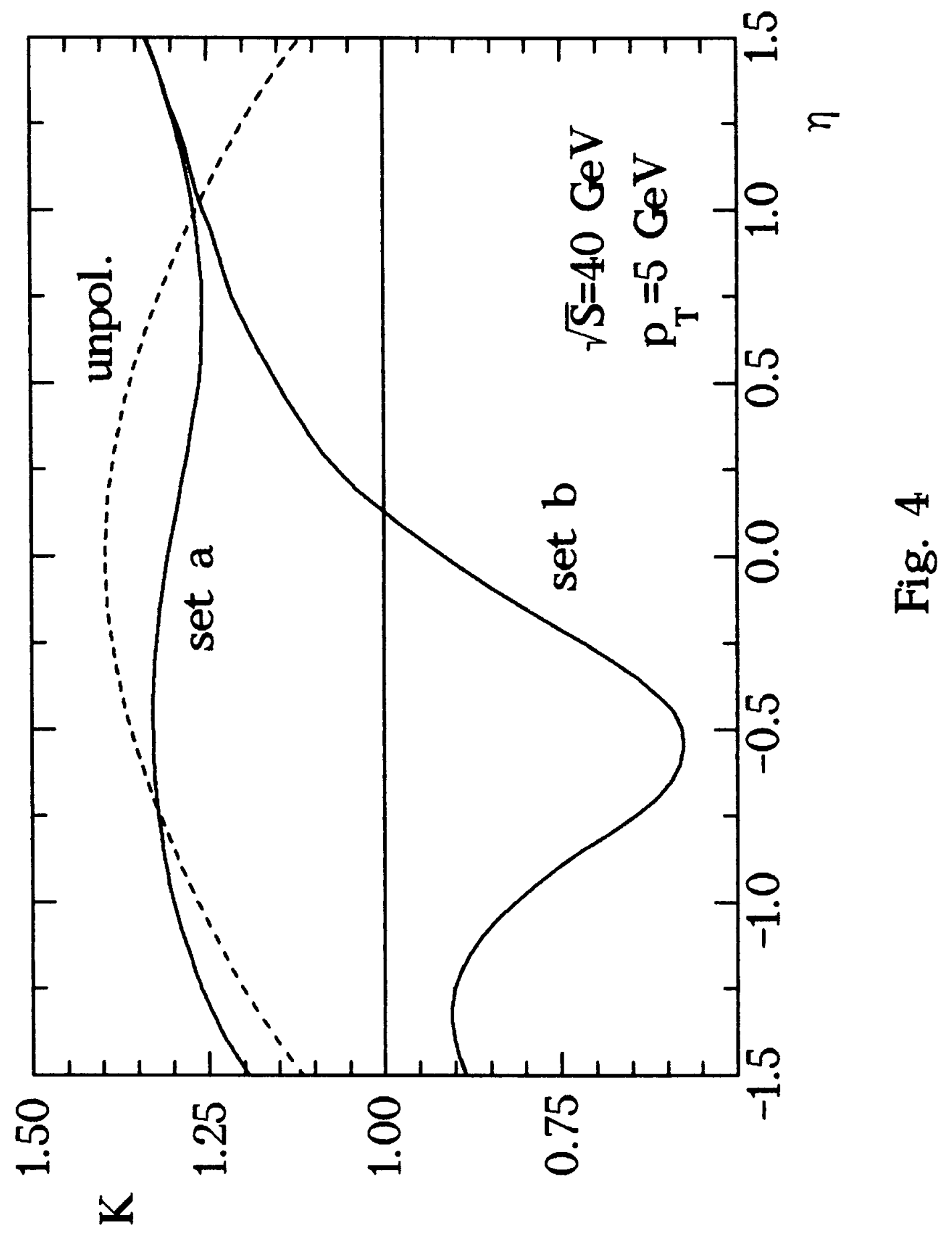




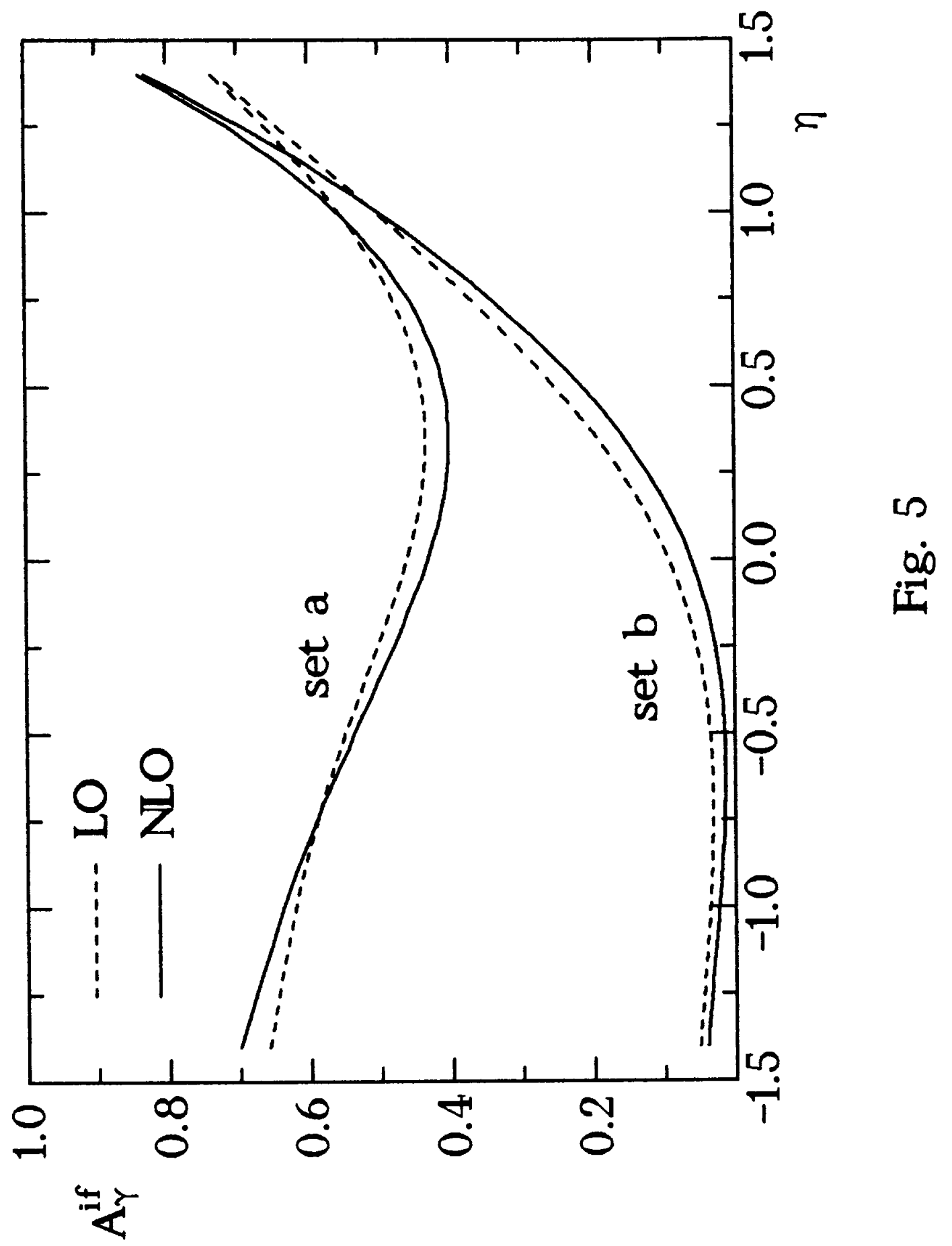




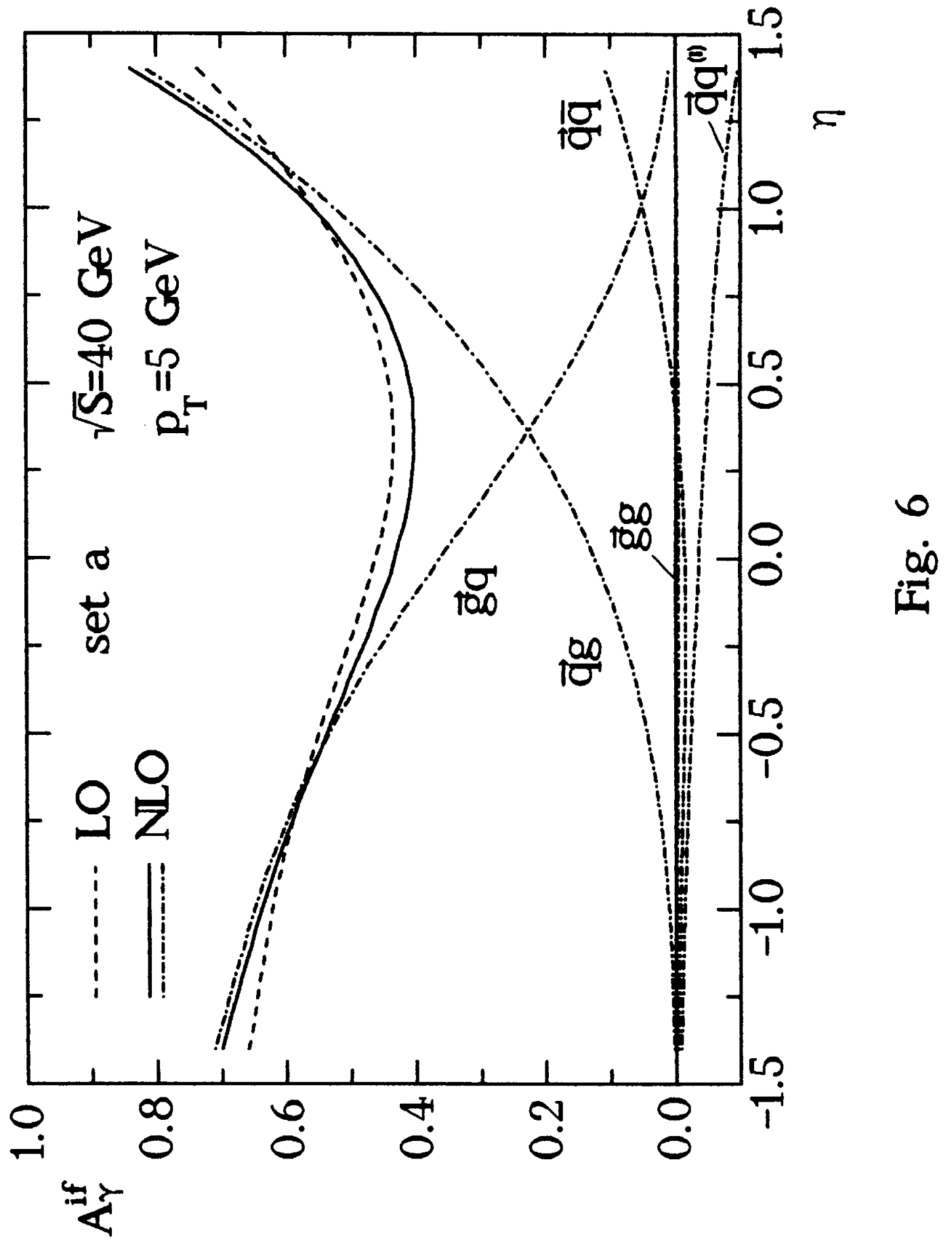




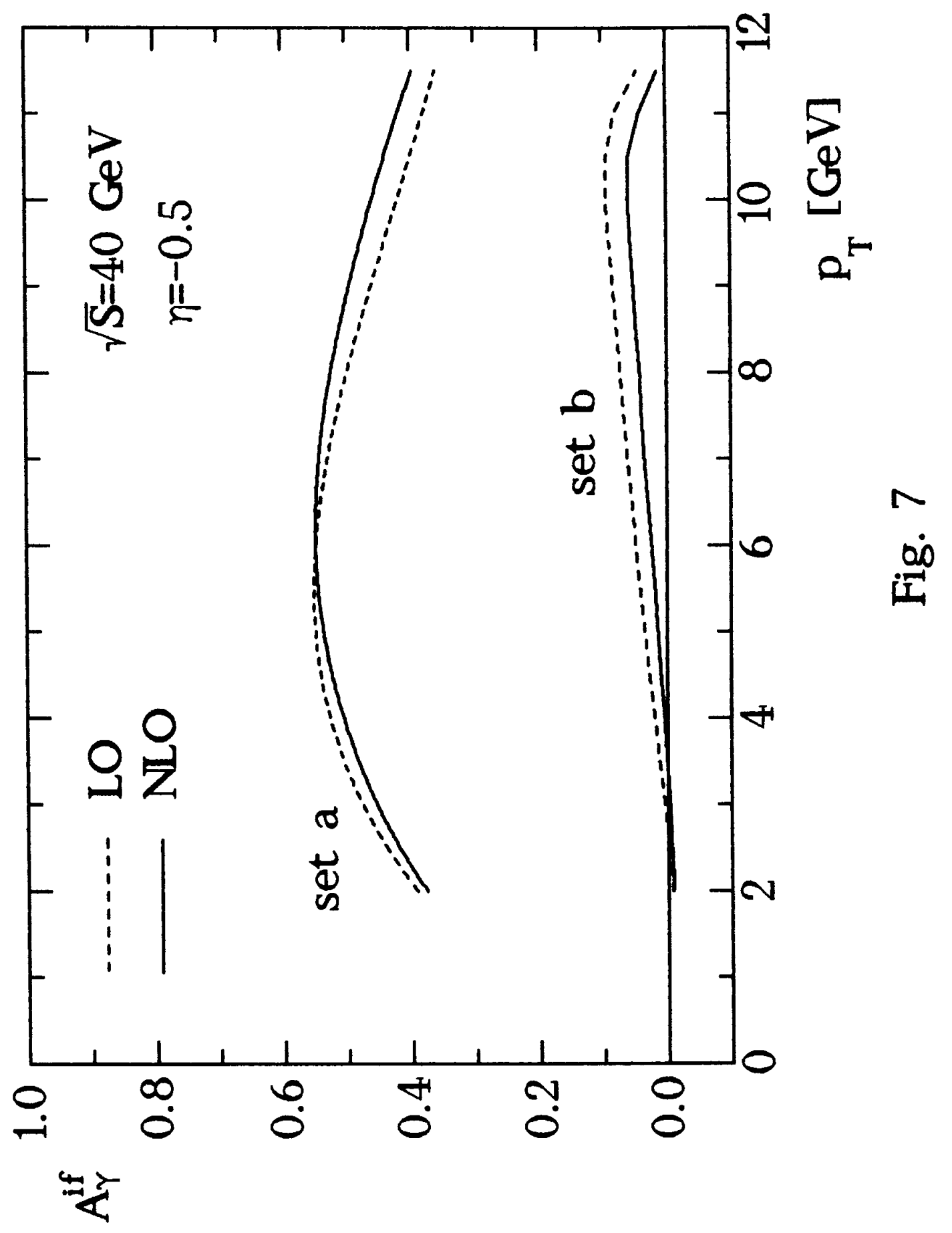




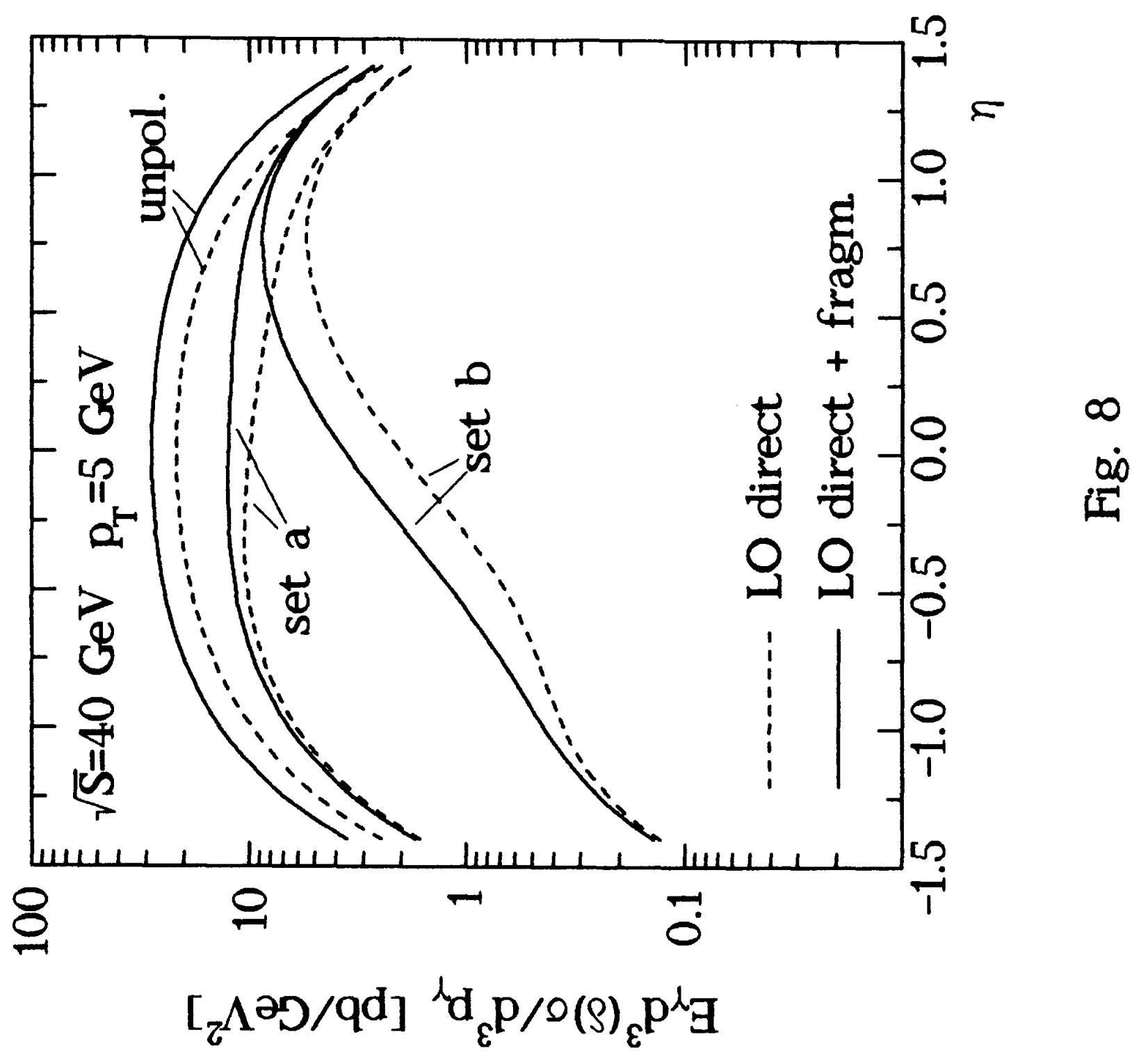




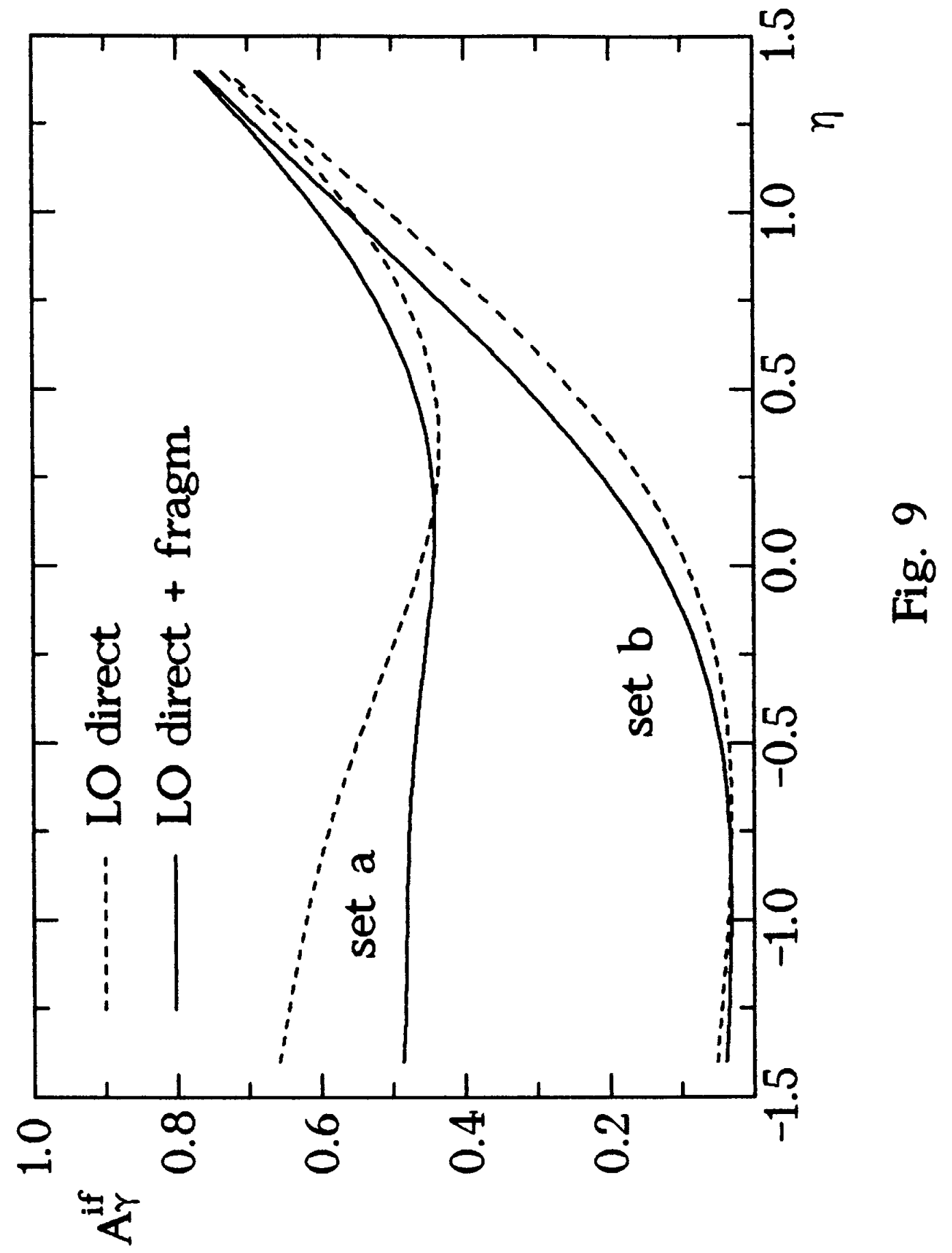




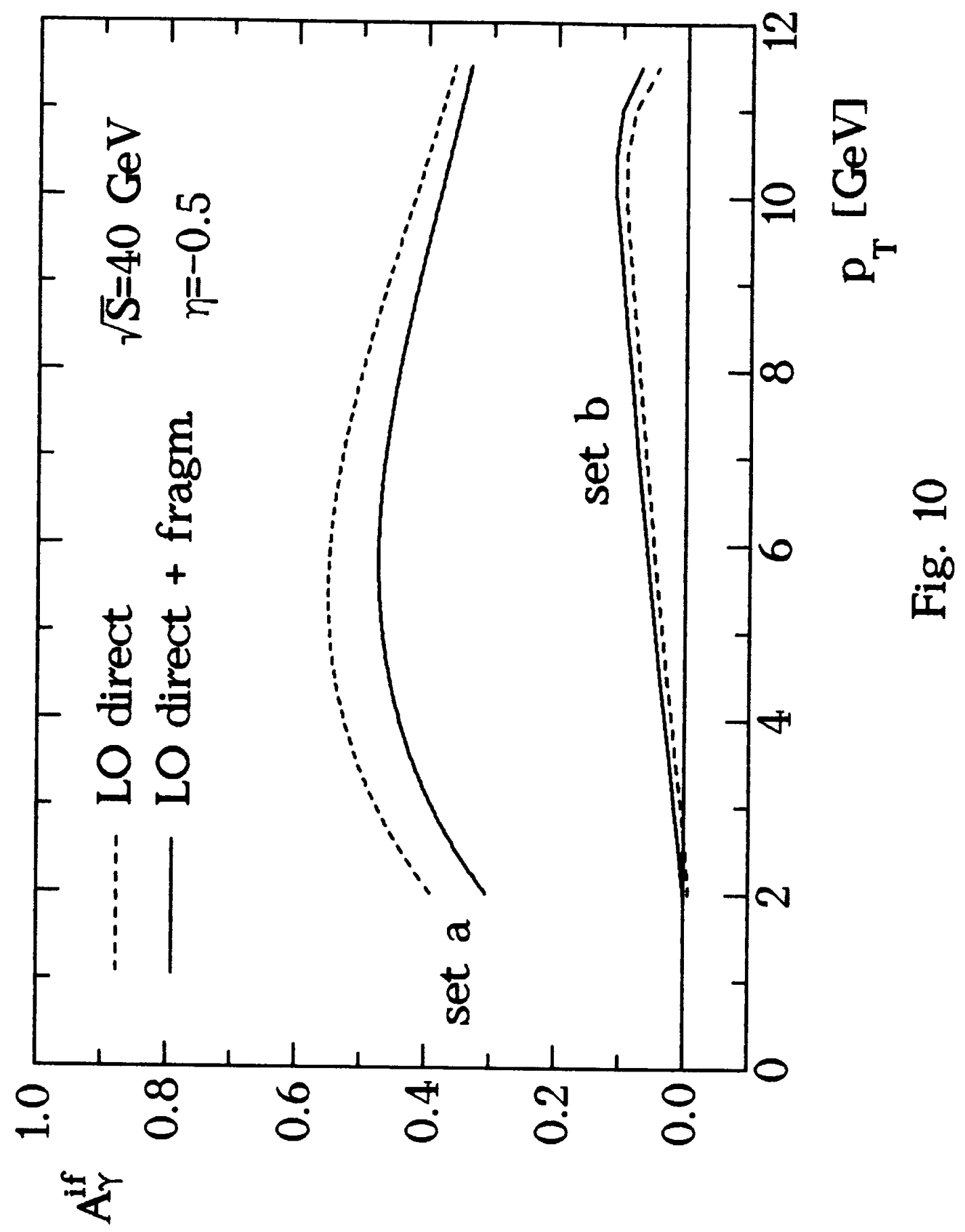

